\title{
Risk Premia and Optimal Liquidation of Defaultable Securities*
}

\author{
Tim Leung ${ }^{\dagger}$ \\ Columbia University
}

\author{
Peng Liu ${ }^{\ddagger}$ \\ Johns Hopkins University
}

Submitted: September 29, 2011

\begin{abstract}
This paper studies the optimal timing to liquidate defaultable securities in a general intensity-based credit risk model under stochastic interest rate. We incorporate the potential price discrepancy between the market and investors, which is characterized by risk-neutral valuation under different default risk premia specifications. To quantify the value of optimally timing to sell, we introduce the delayed liquidation premium which is closely related to the stochastic bracket between the market price and a pricing kernel. We analyze the optimal liquidation policy for various credit derivatives. Our model serves as the building block for the sequential buying and selling problem. We also discuss the extensions to a jump-diffusion default intensity model as well as a defaultable equity model.
\end{abstract}

Keywords: optimal stopping, derivatives liquidation, price discrepancy, default risk premia JEL Classification: G12, G13, C68

${ }^{*}$ This work is partially supported by NSF grant DMS-0908295.

${ }^{\dagger}$ IEOR Department, Columbia University, New York, NY 10027; email: leung@ ieor.columbia.edu.

${ }^{\ddagger}$ Applied Mathematics and Statistics Department, Johns Hopkins University, Baltimore, MD 21218; email: pliu19@jhu.edu. 


\section{Contents}

1 Introduction $\quad 3$

2 Problem Formulation $\quad 4$

2.1 Price Discrepancy . . . . . . . . . . . . . . . . . . . . 4

2.2 Optimal Stopping \& Delayed Liquidation Premium . . . . . . . . . . . . . 5

3 Optimal Liquidation under Markovian Credit Risk Models $\quad 7$

3.1 Pricing Measures and Default Risk Premia . . . . . . . . . . . . . . 7

3.2 Delayed Liquidation Premium and Optimal Timing . . . . . . . . . . . . . . . 9

4 Applications to Defaultable Securities $\quad 11$

4.1 Defaultable Bonds with Zero Recovery . . . . . . . . . . . . . . . . . . . . . 11

4.2 Recovery of Treasury and Market Value . . . . . . . . . . . . . . . . . . . . . 14

4.3 Optimal Liquidation of CDS . . . . . . . . . . . . . . . . . . 15

5 Optimal Buying and Selling $\quad 16$

5.1 Optimal Timing with Short Sale Possibility . . . . . . . . . . . . . 17

5.2 Sequential Buying and Selling . . . . . . . . . . . . . . 17

6 Extensions and Conclusions $\quad 18$

6.1 Jump-Diffusion Default Intensity . . . . . . . . . . . . . . . . . . 18

6.2 Options on a Defaultable Stock . . . . . . . . . . . . . . . 20

6.3 Conclusions . . . . . . . . . . . . . . . . . . . . . 22 


\section{Introduction}

In credit derivatives trading, one important question is how the market compensates investors for bearing credit risk. A number of studies $[2,4,9,18]$ have examined analytically and empirically the structure of default risk premia inferred from the market prices of corporate bonds, credit default swaps, and multi-name credit derivatives. A major risk premium component is the markto-market risk premium which accounts for the fluctuations in default risk. Under reduced-form models of credit risk $[12,19,22]$, this is connected with a drift change of the state variable diffusion driving the default intensity. In addition, there is the event risk premium (or jump-to-default risk premium) that compensates for the uncertain timing of the default event, and is measured by the ratio of the risk-neutral intensity to the historical intensity (see $[2,18]$ ).

From standard no-arbitrage pricing theory, risk premia specification is inherently tied to the selection of risk-neutral pricing measures. A typical buy-side investor (e.g. hedge fund manager or proprietary trader) would identify trading opportunities by looking for mispriced contracts in the market. This can be interpreted as selecting a pricing measure to reflect her view on credit risk evolution and the required risk premia. As a result, the investor's pricing measure may differ from that represented by the prevailing market prices. In a related study, Leung and Ludkovski [23] showed that such a price discrepancy would also arise from pricing under marginal utility.

Price discrepancy is also important for investors with credit-sensitive positions who may need to control risk exposure through liquidation. The central issue lies in the timing of liquidation as investors have the option to sell at the current market price or wait for a later opportunity. The optimal strategy, as we will study, depends on the sources of risks, risk premia, as well as derivative payoffs.

This paper tackles the optimal liquidation problem on two fronts. First, we provide a mathematical framework for price discrepancy between the market and investors under an intensitybased credit risk model. Second, we derive and analyze the optimal stopping problem corresponding to the liquidation of defaultable securities under price discrepancy. As a natural extension, we also examine the optimal buy-and-sell strategy and the associated optimal double-stopping problem.

In order to measure the benefit of optimally timing to sell as opposed to immediate liquidation, we employ the concept of delayed liquidation premium. It turns out to be a very useful tool for analyzing the optimal stopping problem. The intuition is that the investor should wait as long as the delayed liquidation premium is strictly positive. Applying martingale arguments, we deduce the scenarios where immediate or delayed liquidation is optimal (see Theorem 3.4). Moreover, through its probabilistic representation, the delayed liquidation premium reveals the roles of risk premia in the liquidation timing. Under a Markovian credit risk model, the optimal timing is characterized by a liquidation boundary solved from a variational inequality. For numerical illustration, we provide a series of examples where the default intensity and interest rate follow Cox-Ingersoll-Ross (CIR) or Ornstein-Uhlenbeck (OU) processes.

Our study also provides a connection between different risk-neutral pricing measures (or equivalent martingale measures) in incomplete markets. Well-known examples of candidate pricing measures that are consistent with the no-arbitrage principle include the minimal martingale measure [14], the minimal entropy martingale measure [15, 16], and the $q$-optimal martingale measure [17]. For CDO tranche spreads, Cont and Minca [7] constructed a pricing measure and default intensity based on entropy minimization. In this paper, we examine how the choice of pricing measure will influence the investor's liquidation timing.

In recent literature, a number of models have been proposed to incorporate the idea of mispric- 
ing into optimal investment. Cornell et al. [8] studied portfolio optimization based on perceived mispricing from the investor's strong belief in the stock price distribution. Ekström et al. [13] investigated the optimal liquidation of a call spread when the investor's belief on the volatility differs from the implied volatility. Our work is closest in spirit to [24] where the delayed purchase premium concept was used to analyze the optimal timing to buy European and American options under price discrepancy. On the other hand, the problem of optimal liquidation involving price impacts has been studied in [1, 26, 27], among others.

The rest of the paper is organized as follows. In Section 2, we present the mathematical model for price discrepancy and formulate the optimal liquidation problem under a general intensitybased credit risk model. In Section 3, we study the problem within a Markovian market and characterize the optimal liquidation strategy for a general defaultable claim. In Section 4, we apply our analysis to a number of credit derivatives, e.g. defaultable bonds and credit default swaps (CDS). In Section 5, we examine the optimal buy-and-sell strategy for defaultable claims. Section 6 summarizes several extensions and concludes the paper.

\section{Problem Formulation}

This section provides the mathematical formulation of price discrepancy and the optimal liquidation of defaultable securities under an intensity-based credit risk model. We fix a probability space $(\Omega, \mathcal{G}, \mathbb{P})$, where $\mathbb{P}$ is the historical measure, and denote $T$ as the maturity of the defaultable securities. There is a stochastic risk-free interest rate process $\left(r_{t}\right)_{0 \leq t \leq T}$. The default arrival is described by the first jump of a doubly-stochastic Poisson process. Precisely, assuming a default intensity process $\left(\hat{\lambda}_{t}\right)_{0 \leq t \leq T}$, we define the default time $\tau_{d}$ by

$$
\tau_{d}=\inf \left\{t \geq 0: \int_{0}^{t} \hat{\lambda}_{s} d s>E\right\}, \quad \text { where } E \sim \operatorname{Exp}(1) \text { and } E \perp \hat{\lambda}, r .
$$

The associated default counting process is $N_{t}=\mathbf{1}_{\left\{t \geq \tau_{d}\right\}}$. We shall work with two filtrations:

the first one $\mathbb{F}=\left(\mathcal{F}_{t}\right)_{0 \leq t \leq T}$ is generated by $r$ and $\hat{\lambda}$, and the other is the augmented filtration $\mathbb{G}=\left(\mathcal{G}_{t}\right)_{0 \leq t \leq T}$ defined by $\mathcal{G}_{t}=\mathcal{F}_{t} \vee \sigma(E)$.

\subsection{Price Discrepancy}

By standard no-arbitrage pricing theory, the market price of a defaultable claim, denoted by $\left(P_{t}\right)_{0 \leq t \leq T}$, is computed from a conditional expectation of discounted payoff under the market risk-neutral (or equivalent martingale) pricing measure $\mathbb{Q} \sim \mathbb{P}$. In many parametric credit risk models, the market pricing measure $\mathbb{Q}$ is related to the historical measure $\mathbb{P}$ via the default risk premia (see Section 3.1 below). We assume the standard hypothesis $(\mathrm{H})$ that every $\mathbb{F}$-local martingale is a $\mathbb{G}$-local martingale holds under $\mathbb{Q}$ (see $[6, \S 8.3]$ ).

We can describe a general defaultable claim by the quadruple $\left(Y, A, R, \tau_{d}\right)$, where $Y \in \mathcal{F}_{T}$ is the terminal payoff if the defaultable claim survives at $T,\left(A_{t}\right)_{0 \leq t \leq T}$ is a $\mathbb{F}$-adapted continuous process of finite variation with $A_{0}=0$ representing the promised dividends until maturity or default, and $\left(R_{t}\right)_{0 \leq t \leq T}$ is a $\mathbb{F}$-predictable process representing the recovery payoff paid at default. Similar notations are used by Bielecki et al. [5] where the following integrability conditions are 
assumed:

$$
\begin{aligned}
& \mathbb{E}^{\mathbb{Q}}\left\{\left|e^{-\int_{0}^{T} r_{v} d v} Y\right|\right\}<\infty, \quad \mathbb{E}^{\mathbb{Q}}\left\{\left|\int_{(0, T]} e^{-\int_{0}^{u} r_{v} d v}\left(1-N_{u}\right) d A_{u}\right|\right\}<\infty, \quad \text { and } \\
& \mathbb{E}^{\mathbb{Q}}\left\{\left|e^{-\int_{0}^{\tau_{d} \wedge T} r_{v} d v} R_{\tau_{d} \wedge T}\right|\right\}<\infty .
\end{aligned}
$$

For a defaultable claim $\left(Y, A, R, \tau_{d}\right)$, the associated cash flow process $\left(D_{t}\right)_{0 \leq t \leq T}$ is defined by

$$
D_{t}:=Y \mathbf{1}_{\left\{\tau_{d}>T\right\}} \mathbf{1}_{\{t \geq T\}}+\int_{(0, t \wedge T]}\left(1-N_{u}\right) d A_{u}+\int_{(0, t \wedge T]} R_{u} d N_{u}
$$

Then, the (cumulative) market price process $\left(P_{t}\right)_{0 \leq t \leq T}$ under the pricing measure $\mathbb{Q}$ is given by

$$
P_{t}:=\mathbb{E}^{\mathbb{Q}}\left\{\int_{(0, T]} e^{-\int_{t}^{u} r_{v} d v} d D_{u} \mid \mathcal{G}_{t}\right\}
$$

One simple example is the zero-coupon zero-recovery defaultable bond $\left(1,0,0, \tau_{d}\right)$, whose market price is simply $P_{t}=\mathbb{E}^{\mathbb{Q}}\left\{e^{-\int_{t}^{T} r_{v} d v} \mathbf{1}_{\left\{\tau_{d}>T\right\}} \mid \mathcal{G}_{t}\right\}$.

When a perfect replication is unavailable, the market is incomplete and there exist different risk-neutral pricing measures that give different no-arbitrage prices for the same defaultable claim. Mathematically, this amounts to assigning a different risk-neutral pricing measure $\tilde{\mathbb{Q}} \sim \mathbb{Q}$. The investor's reference price process $\left(\tilde{P}_{t}\right)_{0 \leq t \leq T}$ is given by conditional expectation under $\tilde{\mathbb{Q}}$ :

$$
\tilde{P}_{t}:=\mathbb{E}^{\tilde{\mathbb{Q}}}\left\{\int_{(0, T]} e^{-\int_{t}^{u} r_{v} d v} d D_{u} \mid \mathcal{G}_{t}\right\}
$$

whose discounted price process $\left(e^{-\int_{0}^{t} r_{v} d v} \tilde{P}_{t}\right)_{0 \leq t \leq T}$ is a $(\tilde{\mathbb{Q}}, \mathbb{G})$-martingale. We assume that the standard hypothesis $(\mathrm{H})$ also holds under $\tilde{\mathbb{Q}}$.

\subsection{Optimal Stopping \& Delayed Liquidation Premium}

A defaultable claim holder can sell her position at the prevailing market price. If she completely agrees with the market price, then she will be indifferent to sell at any time. Under price discrepancy, however, there is a timing option embedded in the optimal liquidation problem. Precisely, in order to maximize the expected spread between the investor's price and the market price, the holder solves the optimal stopping problem:

$$
J_{t}:=\underset{\tau \in \mathcal{T}_{t, T}}{\operatorname{ess} \sup } \mathbb{E}^{\tilde{\mathbb{Q}}}\left\{e^{-\int_{t}^{\tau} r_{v} d v}\left(P_{\tau}-\tilde{P}_{\tau}\right) \mid \mathcal{G}_{t}\right\}, \quad 0 \leq t \leq T
$$

where $\mathcal{T}_{t, T}$ is the set of $\mathbb{G}$-stopping times taking values in $[t, T]$. Using repeated conditioning, we decompose $(2.6)$ to $J_{t}=V_{t}-\tilde{P}_{t}$, where

$$
V_{t}:=\operatorname{ess}_{\tau \in \mathcal{T}_{t, T}} \mathbb{E}^{\tilde{\mathbb{Q}}}\left\{e^{-\int_{t}^{\tau} r_{v} d v} P_{\tau} \mid \mathcal{G}_{t}\right\} .
$$

Hence, maximizing the price spread in (2.6) is equivalent to maximizing the expected discounted future market value $P_{\tau}$ under the investor's measure $\tilde{\mathbb{Q}}$ in $(2.7)$.

Lemma 2.1. For $0 \leq t \leq T$, we have $V_{t} \geq P_{t} \vee \tilde{P}_{t}$. Also, $V_{\tau_{d}}=\tilde{P}_{\tau_{d}}=P_{\tau_{d}}$ at default. 
Proof. Since $\tau=t$ and $\tau=T$ are candidate liquidation times, it follows that $V_{t} \geq P_{t} \vee \tilde{P}_{t}$. From (2.3) we observe that $P_{t}=\int_{\left(0, \tau_{d}\right]} e^{-\int_{t}^{u} r_{v} d v} d D_{u}=\tilde{P}_{t}$ for $t \geq \tau_{d} \wedge T$. This implies that

$$
V_{\tau_{d}}=\underset{\tau \in \mathcal{T}_{\tau_{d}, T}}{\operatorname{ess} \sup } \mathbb{E}^{\tilde{\mathbb{Q}}}\left\{e^{-\int_{\tau_{d}}^{\tau} r_{v} d v} P_{\tau} \mid \mathcal{G}_{\tau_{d}}\right\}=\operatorname{ess~sup}_{\tau \in \mathcal{T}_{\tau_{d}, T}} \mathbb{E}^{\tilde{\mathbb{Q}}}\left\{e^{-\int_{\tau_{d}}^{\tau} r_{v} d v} \tilde{P}_{\tau} \mid \mathcal{G}_{\tau_{d}}\right\}=\tilde{P}_{\tau_{d}}=P_{\tau_{d}}
$$

The last equation means that price discrepancy vanishes when the default event is observed or when the contract expires. This is also realistic since the market will no longer be liquid afterward.

According to (2.7), the optimal liquidation timing directly depends on the investor's pricing measure $\tilde{\mathbb{Q}}$ as well as the market $\mathbb{Q}$ (via the market price $P$ ). Specifically, we observe that the discounted market price $\left(e^{-\int_{0}^{t} r_{v} d v} P_{t}\right)_{0 \leq t \leq T}$ is a $(\mathbb{Q}, \mathbb{G})$-martingale, but generally not a $(\tilde{\mathbb{Q}}, \mathbb{G})$ martingale. If the discounted market price is a $(\tilde{\mathbb{Q}}, \mathbb{G})$-supermartingale, then it is optimal to sell the claim immediately. If the discounted market price turns out to be a $(\tilde{\mathbb{Q}}, \mathbb{G})$-submartingale, then it is optimal to delay the liquidation until maturity $T$. Besides these two scenarios, the optimal liquidation strategy may be non-trivial.

To quantify the value of optimally waiting to sell, we define the delayed liquidation premium:

$$
L_{t}:=V_{t}-P_{t} \geq 0
$$

It is often more intuitive to study the optimal liquidation timing in terms of the premium $L$. Indeed, standard optimal stopping theory [21, Appendix D] suggests that the optimal stopping time $\tau^{*}$ for $(2.7)$ is the first time the process $V$ reaches the reward $P$, namely,

$$
\tau^{*}=\inf \left\{t \leq u \leq T: V_{u}=P_{u}\right\}=\inf \left\{t \leq u \leq T: L_{u}=0\right\} .
$$

The last equation, which follows directly from definition (2.9), implies that the investor will liquidate as soon as the delayed liquidation premium vanishes. Moreover, we also observe from (2.8) and (2.10) that $\tau^{*} \leq \tau_{d}$. Next, we express $L$ as an optimal stopping problem.

Lemma 2.2. The delayed liquidation premium $L$ admits the probabilistic representation:

$$
L_{t}=Z_{t}^{-1} \underset{\tau \in \mathcal{T}_{t, T}}{\operatorname{ess} \sup } \mathbb{E}^{\mathbb{Q}}\left\{\int_{t}^{\tau} e^{-\int_{t}^{u} r_{v} d v} d[P, Z]_{u} \mid \mathcal{G}_{t}\right\},
$$

where $Z_{t}:=\mathbb{E}^{\mathbb{Q}}\left\{\frac{d \tilde{\mathbb{Q}}}{d \mathbb{Q}} \mid \mathcal{G}_{t}\right\}$ is the density process of $\tilde{\mathbb{Q}}$ with respect to $\mathbb{Q}$.

Proof. Denote the discounted market price by $\hat{P}_{u}:=e^{-\int_{0}^{u} r_{v} d v} P_{u}$. Under measure $\mathbb{Q}$, the product $Z \hat{P}$ satisfies the SDE:

$$
Z_{\tau} \hat{P}_{\tau}=Z_{t} \hat{P}_{t}+\int_{t}^{\tau} Z_{u-} d \hat{P}_{u}+\int_{t}^{\tau} \hat{P}_{u_{-}} d Z_{u}+\int_{t}^{\tau} d[\hat{P}, Z]_{u}
$$

By definition and the integrability condition $(2.2)$, both $\hat{P}$ and $Z$ are $(\mathbb{Q}, \mathbb{G})$-martingales. Applying this and (2.12) to (2.9), we get

$$
L_{t}=Z_{t}^{-1} e^{\int_{0}^{t} r_{v} d v} \underset{\tau \in \mathcal{T}_{t, T}}{\operatorname{ess} \sup _{1}} \mathbb{E}^{\mathbb{Q}}\left\{\hat{P}_{\tau} Z_{\tau} \mid \mathcal{G}_{t}\right\}-P_{t}=Z_{t}^{-1} \underset{\tau \in \mathcal{T}_{t, T}}{\operatorname{ess} \sup } \mathbb{E}^{\mathbb{Q}}\left\{\int_{t}^{\tau} e^{-\int_{t}^{u} r_{v} d v} d[P, Z]_{u} \mid \mathcal{G}_{t}\right\} .
$$


In particular, the delayed liquidation premium depends on the product of the pricing kernel $Z_{u}$ (between $\tilde{\mathbb{Q}}$ and $\mathbb{Q}$ ) and the market price $P_{u}$. From $(2.11)$, the stochastic bracket $[P, Z]$ plays a vital role in the analysis of $L$ and $\tau^{*}$. This is especially useful under some parametric credit risk models where $[P, Z]$ is explicit.

\section{Optimal Liquidation under Markovian Credit Risk Models}

We proceed to analyze the optimal liquidation problem under a general class of Markovian credit risk models. The description of various pricing measures will involve the mark-to-market risk premium and event risk premium, which are crucial in the characterization of the optimal liquidation strategy (see Theorem 3.4).

\subsection{Pricing Measures and Default Risk Premia}

We consider a $n$-dimensional Markovian state vector process $\mathbf{X}$ that drives the interest rate $r_{t}=r\left(t, \mathbf{X}_{t}\right)$ and default intensity $\hat{\lambda}_{t}=\hat{\lambda}\left(t, \mathbf{X}_{t}\right)$ for some positive measurable functions $r(\cdot, \cdot)$ and $\hat{\lambda}(\cdot, \cdot)$. Denote by $\mathbb{F}$ the filtration generated by $\mathbf{X}$. We also assume a Markovian payoff structure for the defaultable claim $\left(Y, A, R, \tau_{d}\right)$ with $Y=Y\left(\mathbf{X}_{T}\right), A_{t}=\int_{0}^{t} q\left(u, \mathbf{X}_{u}\right) d u$, and $R_{t}=R\left(t, \mathbf{X}_{t}\right)$ for some measurable functions $Y(\cdot), q(\cdot, \cdot)$, and $R(\cdot, \cdot)$ satisfying integrability conditions $(2.2)$.

Under the historical measure $\mathbb{P}$, the state vector process $\mathbf{X}$ satisfies the SDE

$$
d \mathbf{X}_{t}=a\left(t, \mathbf{X}_{t}\right) d t+\Sigma\left(t, \mathbf{X}_{t}\right) d \mathbf{W}_{t}^{\mathbb{P}}
$$

where $\mathbf{W}^{\mathbb{P}}$ is a $m$-dimensional $\mathbb{P}$-Brownian motion, $a$ is the deterministic drift function, and $\Sigma$ is the $n$ by $m$ deterministic volatility function. Standard Lipschitz and growth conditions [20, §5.2] are assumed to guarantee a unique solution to (3.1).

Applying multi-dimensional Girsanov Theorem for the market pricing measure $\mathbb{Q} \sim \mathbb{P}$, there exists an adapted process $\left(\boldsymbol{\phi}_{t}^{\mathbb{Q}, \mathbb{P}}\right)_{0 \leq t \leq T}$ such that $\mathbf{W}_{t}^{\mathbb{Q}}=\mathbf{W}_{t}^{\mathbb{P}}+\int_{0}^{t} \boldsymbol{\phi}_{u}^{\mathbb{Q}, \mathbb{P}} d u$ is a $m$-dimensional $\mathbb{Q}$ Brownian motion. The process $\phi^{\mathbb{Q}, \mathbb{P}}$ is commonly referred to as the mark-to-market risk premium (see [2]), which is assumed herein to be Markovian of the form $\phi^{\mathbb{Q}, \mathbb{P}}\left(t, \mathbf{X}_{t}\right)$. The $\mathbb{Q}$-dynamics of $\mathbf{X}$ are given by

$$
d \mathbf{X}_{t}=b\left(t, \mathbf{X}_{t}\right) d t+\Sigma\left(t, \mathbf{X}_{t}\right) d \mathbf{W}_{t}^{\mathbb{Q}}
$$

where $b\left(t, \mathbf{X}_{t}\right):=a\left(t, \mathbf{X}_{t}\right)-\Sigma\left(t, \mathbf{X}_{t}\right) \boldsymbol{\phi}^{\mathbb{Q}, \mathbb{P}}\left(t, \mathbf{X}_{t}\right)$.

In addition, the holder is subject to the risk from the uncertain timing of default. The associated event risk premium (see $[2,18]$ ) is defined by the ratio between the $\mathbb{Q}$-intensity and the $\mathbb{P}$-intensity, namely, $\mu\left(t, \mathbf{X}_{t}\right)=\lambda\left(t, \mathbf{X}_{t}\right) / \hat{\lambda}\left(t, \mathbf{X}_{t}\right)$.

Similarly, the investor's pricing measure $\tilde{\mathbb{Q}}$ is related to the historical measure $\mathbb{P}$ through the investor's Markovian risk premium functions $\boldsymbol{\phi}^{\tilde{\mathbb{Q}}, \mathbb{P}}(t, \mathbf{x})$ and $\tilde{\mu}(t, \mathbf{x})$. By a change of measure, the drift of $\mathbf{X}$ under $\tilde{\mathbb{Q}}$ is modified to $\tilde{b}\left(t, \mathbf{X}_{t}\right):=a\left(t, \mathbf{X}_{t}\right)-\Sigma\left(t, \mathbf{X}_{t}\right) \boldsymbol{\phi}^{\tilde{\mathbb{Q}}, \mathbb{P}}\left(t, \mathbf{X}_{t}\right)$.

Define $M_{t}^{\mathbb{Q}}=N_{t}-\int_{0}^{t} \lambda_{u}\left(1-N_{u}\right) d u$ as the compensated $(\mathbb{Q}, \mathbb{G})$-martingale associated with $N$. Then, the EMMs $\mathbb{Q}$ and $\tilde{\mathbb{Q}}$ are related by the Radon-Nikodym derivative:

$$
Z_{t}=\frac{d \tilde{\mathbb{Q}}}{d \mathbb{Q}} \mid \mathcal{G}_{t}=\mathcal{E}\left(-\phi^{\tilde{\mathbb{Q}}, \mathbb{Q}} \cdot \mathbf{W}^{\mathbb{Q}}\right)_{t} \mathcal{E}\left(\frac{\tilde{\mu}}{\mu} M^{\mathbb{Q}}\right)_{t}
$$


where the Doléans-Dade exponentials are defined by

$$
\begin{aligned}
\mathcal{E}\left(-\boldsymbol{\phi}^{\tilde{\mathbb{Q}}, \mathbb{Q}} \cdot \mathbf{W}^{\mathbb{Q}}\right)_{t} & :=\exp \left(-\frac{1}{2} \int_{0}^{t}\left\|\boldsymbol{\phi}_{u}^{\tilde{\mathbb{Q}}, \mathbb{Q}}\right\|^{2} d u-\int_{0}^{t} \boldsymbol{\phi}_{u}^{\tilde{\mathbb{Q}}, \mathbb{Q}} \cdot d \mathbf{W}_{u}^{\mathbb{Q}}\right) \\
\mathcal{E}\left(\frac{\tilde{\mu}}{\mu} M^{\mathbb{Q}}\right)_{t} & :=\exp \left(\int_{0}^{t} \log \left(\frac{\tilde{\mu}_{u-}}{\mu_{u-}}\right) d N_{u}-\int_{0}^{t}\left(1-N_{u}\right)\left(\tilde{\mu}_{u}-\mu_{u}\right) \hat{\lambda}_{u} d u\right) .
\end{aligned}
$$

We assume that $\phi^{\tilde{\mathbb{Q}}, \mathbb{Q}}$ is Markovian satisfying $\int_{0}^{T}\left\|\boldsymbol{\phi}_{u}^{\tilde{\mathbb{Q}}, \mathbb{Q}}\right\|^{2} d u<\infty$, and $\int_{0}^{T}\left|\tilde{\mu}_{u}-\mu_{u}\right| \hat{\lambda}_{u} d u<\infty$ (see Theorem 4.8 of [28]). From the decomposition:

$$
\boldsymbol{\phi}_{t}^{\tilde{\mathbb{Q}}, \mathbb{Q}} d t=d \mathbf{W}_{t}^{\tilde{\mathbb{Q}}}-d \mathbf{W}_{t}^{\mathbb{Q}}=\left(d \mathbf{W}_{t}^{\tilde{\mathbb{Q}}}-d \mathbf{W}_{t}^{\mathbb{P}}\right)-\left(d \mathbf{W}_{t}^{\mathbb{Q}}-d \mathbf{W}_{t}^{\mathbb{P}}\right)=\left(\boldsymbol{\phi}_{t}^{\tilde{\mathbb{Q}}, \mathbb{P}}-\boldsymbol{\phi}_{t}^{\mathbb{Q}, \mathbb{P}}\right) d t
$$

we can interpret $\phi^{\tilde{\mathbb{Q}}, \mathbb{Q}}$ as the incremental mark-to-market risk premium assigned by the investor relative to the market. On the other hand, the discrepancy in event risk premia is accounted for in the second Doléans-Dade exponential (3.5).

Example 3.1. The OU Model. Suppose $(r, \hat{\lambda})=\mathbf{X}$, following the OU dynamics:

$$
\left(\begin{array}{l}
d r_{t} \\
d \hat{\lambda}_{t}
\end{array}\right)=\left(\begin{array}{c}
\hat{\kappa}_{r}\left(\hat{\theta}_{r}-r_{t}\right) \\
\hat{\kappa}_{\lambda}\left(\hat{\theta}_{\lambda}-\hat{\lambda}_{t}\right)
\end{array}\right) d t+\left(\begin{array}{cc}
\sigma_{r} & 0 \\
\sigma_{\lambda} \rho & \sigma_{\lambda} \sqrt{1-\rho^{2}}
\end{array}\right)\left(\begin{array}{l}
d W_{t}^{1, \mathbb{P}} \\
d W_{t}^{2, \mathbb{P}}
\end{array}\right),
$$

with constant parameters $\hat{\kappa}_{r}, \hat{\theta}_{r}, \hat{\kappa}_{\lambda}, \hat{\theta}_{\lambda} \geq 0$. Here, $\hat{\kappa}_{r}, \hat{\kappa}_{\lambda}$ parameterize the speed of mean reversion, and $\hat{\theta}_{r}, \hat{\theta}_{\lambda}$ represent the long-term means (see [28, §7.1.1]). Assuming a constant event risk premium $\mu$ by the market, the $\mathbb{Q}$-intensity is specified by $\lambda_{t}=\mu \hat{\lambda}_{t}$ and the pair $(r, \lambda)$ satisfies SDEs:

$$
\left(\begin{array}{l}
d r_{t} \\
d \lambda_{t}
\end{array}\right)=\left(\begin{array}{c}
\kappa_{r}\left(\theta_{r}-r_{t}\right) \\
\kappa_{\lambda}\left(\mu \theta_{\lambda}-\lambda_{t}\right)
\end{array}\right) d t+\left(\begin{array}{cc}
\sigma_{r} & 0 \\
\mu \sigma_{\lambda} \rho & \mu \sigma_{\lambda} \sqrt{1-\rho^{2}}
\end{array}\right)\left(\begin{array}{l}
d W_{t}^{1, \mathbb{Q}} \\
d W_{t}^{2, \mathbb{Q}}
\end{array}\right)
$$

with constants $\kappa_{r}, \theta_{r}, \kappa_{\lambda}, \theta_{\lambda} \geq 0$. Under the investor's measure $\tilde{\mathbb{Q}}$, the SDEs for $r_{t}$ and $\tilde{\lambda}_{t}=\tilde{\mu} \hat{\lambda}_{t}$ are of the same form with parameters $\tilde{\kappa}_{r}, \tilde{\theta}_{r}, \tilde{\kappa}_{\lambda}, \tilde{\theta}_{\lambda}$ and $\tilde{\mu}$, and $\mathbf{W}^{\mathbb{Q}}$ is replaced by $\mathbf{W}^{\tilde{\mathbb{Q}}}$.

Direct computation yields the relative mark-to-market risk premium:

$$
\phi_{t}^{\tilde{\mathbb{Q}}, \mathbb{Q}}=\left(\begin{array}{c}
\frac{\kappa_{r}\left(\theta_{r}-r_{t}\right)-\tilde{\kappa}_{r}\left(\tilde{\theta}_{r}-r_{t}\right)}{\sigma_{r}} \\
\frac{1}{\sqrt{1-\rho^{2}}} \frac{\kappa_{\lambda}\left(\theta_{\lambda}-\hat{\lambda}_{t}\right)-\tilde{\kappa}_{\lambda}\left(\tilde{\theta}_{\lambda}-\hat{\lambda}_{t}\right)}{\sigma_{\lambda}}-\frac{\rho}{\sqrt{1-\rho^{2}}} \frac{\kappa_{r}\left(\theta_{r}-r_{t}\right)-\tilde{\kappa}_{r}\left(\tilde{\theta}_{r}-r_{t}\right)}{\sigma_{r}}
\end{array}\right) .
$$

The upper term is the incremental risk premium for the interest rate while the bottom term reflects the discrepancy in the default risk premia (see (3.6)).

Example 3.2. The CIR Model. Let $\mathbf{X}=\left(X^{1}, \ldots, X^{n}\right)$ follow the multifactor CIR model $[28, \S 7.2]$ :

$$
d X_{t}^{i}=\hat{\kappa}_{i}\left(\hat{\theta}_{i}-X_{t}^{i}\right) d t+\sigma_{i} \sqrt{X_{t}^{i}} d W_{t}^{i, \mathbb{P}}
$$

where $W^{i, \mathbb{P}}$ are mutually independent $\mathbb{P}$-Brownian motions and $\hat{\kappa}_{i}, \hat{\theta}_{i}, \sigma_{i} \geq 0, i=1, \ldots, n$ satisfy Feller condition $2 \hat{\kappa}_{i} \hat{\theta}_{i}>\sigma_{i}^{2}$. The interest rate $r$ and historical default intensity $\hat{\lambda}$ are non-negative linear combinations of $X^{i}$ with constant weights $w_{i}^{r}, w_{i}^{\lambda} \geq 0$, namely, $r_{t}=\sum_{i=1}^{n} w_{i}^{r} X_{t}^{i}$ 
and $\hat{\lambda}_{t}=\sum_{i=1}^{n} w_{i}^{\lambda} X_{t}^{i}$. Under measure $\mathbb{Q}, X^{i}$ satisfies the SDE:

$$
d X_{t}^{i}=\kappa_{i}\left(\theta_{i}-X_{t}^{i}\right) d t+\sigma_{i} \sqrt{X_{t}^{i}} d W_{t}^{i, \mathbb{Q}},
$$

with new mean reversion speed $\kappa_{i}$ and long-run mean $\theta_{i}$.

Under the investor's measure $\tilde{\mathbb{Q}}$, the SDE for the state vector is of the same form with new parameters $\tilde{\kappa}_{i}, \tilde{\theta}_{i}$. The associated relative mark-to-market risk premium has following structure:

$$
\phi_{i, t}^{\tilde{\mathbb{Q}}, \mathbb{Q}}=\frac{\kappa_{i}\left(\theta_{i}-X_{t}^{i}\right)-\tilde{\kappa}_{i}\left(\tilde{\theta}_{i}-X_{t}^{i}\right)}{\sigma_{i} \sqrt{X_{t}^{i}}} .
$$

The event risk premia $(\mu, \tilde{\mu})$ are assigned via $\lambda_{t}=\mu \hat{\lambda}_{t}$ under $\mathbb{Q}$ and $\tilde{\lambda}_{t}=\tilde{\mu} \hat{\lambda}_{t}$ under $\tilde{\mathbb{Q}}$ respectively. Remark 3.3. The current framework can be readily generalized to the situation where the investor needs to assume an alternative historical measure $\tilde{\mathbb{P}}$. The resulting risk premium $\phi^{\tilde{\mathbb{Q}}, \mathbb{Q}}$ will have a third decomposition component $\phi^{\tilde{\mathbb{P}}, \mathbb{P}}$, reflecting the difference in historical dynamics.

For any defaultable claim $\left(Y, A, R, \tau_{d}\right)$, the ex-dividend pre-default market price is given by

$$
C\left(t, \mathbf{X}_{t}\right)=\mathbb{E}^{\mathbb{Q}}\left\{e^{-\int_{t}^{T}\left(r_{v}+\lambda_{v}\right) d v} Y\left(\mathbf{X}_{T}\right)+\int_{t}^{T} e^{-\int_{t}^{u}\left(r_{v}+\lambda_{v}\right) d v}\left(\lambda_{u} R\left(u, \mathbf{X}_{u}\right)+q\left(u, \mathbf{X}_{u}\right)\right) d u \mid \mathcal{F}_{t}\right\} .
$$

The associated cumulative price is related to the pre-default price via

$$
P_{t}=\left(1-N_{t}\right) C\left(t, \mathbf{X}_{t}\right)+\int_{0}^{t}\left(1-N_{u}\right) q\left(u, \mathbf{X}_{u}\right) e^{\int_{u}^{t} r_{v} d v} d u+\int_{(0, t]} R\left(u, \mathbf{X}_{u}\right) e^{\int_{u}^{t} r_{v} d v} d N_{u}
$$

The price function $C(t, \mathbf{x})$ can be determined by solving the PDE:

$$
\left\{\begin{array}{l}
\frac{\partial C}{\partial t}(t, \mathbf{x})+\mathcal{L}_{b, \lambda} C(t, \mathbf{x})+\lambda(t, \mathbf{x}) R(t, \mathbf{x})+q(t, \mathbf{x})=0, \quad(t, \mathbf{x}) \in[0, T) \times \mathbb{R}^{n}, \\
C(T, \mathbf{x})=Y(\mathbf{x}), \quad \mathbf{x} \in \mathbb{R}^{n},
\end{array}\right.
$$

where $\mathcal{L}_{x}$ is the operator defined by

$$
\mathcal{L}_{b, \lambda} f=\sum_{i=1}^{n} b_{i}(t, \mathbf{x}) \frac{\partial f}{\partial x_{i}}+\frac{1}{2} \sum_{i, j=1}^{n}\left(\Sigma(t, \mathbf{x}) \Sigma(t, \mathbf{x})^{T}\right)_{i j} \frac{\partial^{2} f}{\partial x_{i} \partial x_{j}}-(r(t, \mathbf{x})+\lambda(t, \mathbf{x})) f .
$$

The computation is similar for the investor's price under $\tilde{\mathbb{Q}}$.

\subsection{Delayed Liquidation Premium and Optimal Timing}

Next, we analyze the optimal liquidation problem $V$ defined in (2.7) for the general defaultable claim under the current Markovian setting.

Theorem 3.4. For a general defaultable claim $\left(Y, A, R, \tau_{d}\right)$ under the Markovian credit risk model, the delayed liquidation premium admits the probabilistic representation:

$$
L_{t}=\mathbf{1}_{\left\{t<\tau_{d}\right\}} \underset{\tau \in \mathcal{T}_{t, T}}{\operatorname{ess} \sup } \mathbb{E}^{\tilde{\mathbb{Q}}}\left\{\int_{t}^{\tau} e^{-\int_{t}^{u}\left(r_{v}+\tilde{\lambda}_{v}\right) d v} G\left(u, \mathbf{X}_{u}\right) d u \mid \mathcal{F}_{t}\right\}
$$


where $G:[0, T] \times \mathbb{R}^{n} \mapsto \mathbb{R}$ is defined by

$$
G(t, \mathbf{x})=-\left(\nabla_{x} C(t, \mathbf{x})\right)^{T} \Sigma(t, \mathbf{x}) \boldsymbol{\phi}^{\tilde{\mathbb{Q}}, \mathbb{Q}}(t, \mathbf{x})+(R(t, \mathbf{x})-C(t, \mathbf{x}))(\tilde{\mu}(t, \mathbf{x})-\mu(t, \mathbf{x})) \hat{\lambda}(t, \mathbf{x}) .
$$

If $G(t, \mathbf{x}) \geq 0 \forall(t, \mathbf{x})$, then it is optimal to delay the liquidation till maturity $T$.

If $G(t, \mathbf{x}) \leq 0 \forall(t, \mathbf{x})$, then it is optimal to sell immediately.

Proof. Using Lemma 2.2, we need to compute $[P, Z]$. Following from (3.12) and (3.3), we get

$$
d P_{u} d Z_{u}=Z_{u-}\left(\left(1-N_{u}\right) G\left(u, \mathbf{X}_{u}\right) d u+\left(\frac{\tilde{\mu}\left(u, \mathbf{X}_{u}\right)}{\mu\left(u, \mathbf{X}_{u}\right)}-1\right)\left(R\left(u, \mathbf{X}_{u}\right)-C\left(u, \mathbf{X}_{u}\right)\right) d M_{u}^{\mathbb{Q}}\right),
$$

where $G$ is defined in (3.16). Applying Lemma 2.2 yields

$$
\begin{aligned}
L_{t} & =Z_{t}^{-1} \operatorname{esssup}_{\tau \in \mathcal{T}_{t, T}} \mathbb{E}^{\mathbb{Q}}\left\{\int_{t}^{\tau} Z_{u}\left(1-N_{u}\right) e^{-\int_{t}^{u} r_{v} d v} G\left(u, \mathbf{X}_{u}\right) d u \mid \mathcal{G}_{t}\right\} \\
& =\underset{\tau \in \mathcal{T}_{t, T}}{\operatorname{ess} \sup _{t}} \mathbb{E}^{\tilde{\mathbb{Q}}}\left\{\int_{t}^{\tau}\left(1-N_{u}\right) e^{-\int_{t}^{u} r_{v} d v} G\left(u, \mathbf{X}_{u}\right) d u \mid \mathcal{G}_{t}\right\} .
\end{aligned}
$$

Then, (3.15) follows from the standard change of filtration [6, §5.1.1]. If $G \geq 0$, then the integrand in (3.15) is positive a.s. and therefore the largest possible stopping time $T$ is optimal. If $G \leq 0$, then $\tau^{*}=t$ is optimal and $L_{t}=0$ a.s.

The drift function $G$ has two components explicitly depending on $\phi^{\tilde{\mathbb{Q}}, \mathbb{Q}}$ and $\tilde{\mu}-\mu$. If $\phi^{\tilde{\mathbb{Q}}, \mathbb{Q}}(t, \mathbf{x})=\mathbf{0} \forall(t, \mathbf{x})$, that is, the investor and market agree on the mark-to-market risk premium, then the sign of $G$ is solely determined by the difference $\tilde{\mu}-\mu$, since recovery $R$ in general is less than the pre-default price $C$. On the other hand, if $\mu(t, \mathbf{x})=\tilde{\mu}(t, \mathbf{x}) \forall(t, \mathbf{x})$, then the second term of $G$ vanishes but $G$ still depends on $\mu$ through $\nabla_{x} C$ in the first term. In the special example with constant $\lambda$ and $r$, the first term of drift function in (3.16) vanishes, but the second term remains due to potential discrepancy in event risk premium.

When the drift function is not of constant sign, the optimal liquidation policy may be nontrivial and needs to be numerically determined. For this purpose, we write $L_{t}=\mathbf{1}_{\left\{t<\tau_{d}\right\}} \hat{L}\left(t, \mathbf{X}_{t}\right)$, where $\hat{L}$ is the (Markovian) pre-default delayed liquidation premium defined by

$$
\hat{L}\left(t, \mathbf{X}_{t}\right)=\operatorname{esssup}_{\tau \in \mathcal{T}_{t, T}} \mathbb{E}^{\tilde{\mathbb{Q}}}\left\{\int_{t}^{\tau} e^{-\int_{t}^{u}\left(r_{v}+\tilde{\lambda}_{v}\right) d v} G\left(u, \mathbf{X}_{u}\right) d u \mid \mathcal{F}_{t}\right\}
$$

We determine $\hat{L}$ from the variational inequality:

$$
\min \left(-\frac{\partial \hat{L}}{\partial t}(t, \mathbf{x})-\mathcal{L}_{\tilde{b}, \tilde{\lambda}} \hat{L}(t, \mathbf{x})-G(t, \mathbf{x}), \hat{L}(t, \mathbf{x})\right)=0, \quad(t, \mathbf{x}) \in[0, T) \times \mathbb{R}^{n}
$$

where $\mathcal{L}_{\tilde{b}, \tilde{\lambda}}$ is defined in (3.14), and the terminal condition is $\hat{L}(T, \mathbf{x})=0$, for $\mathbf{x} \in \mathbb{R}^{n}$.

The investor's optimal timing is characterized by the sell region $\mathcal{S}$ and delay region $\mathcal{D}$, namely,

$$
\begin{aligned}
& \mathcal{S}=\left\{(t, \mathbf{x}) \in[0, T] \times \mathbb{R}^{n}: \hat{L}(t, \mathbf{x})=0\right\}, \\
& \mathcal{D}=\left\{(t, \mathbf{x}) \in[0, T] \times \mathbb{R}^{n}: \hat{L}(t, \mathbf{x})>0\right\} .
\end{aligned}
$$

Also, define $\hat{\tau}^{*}=\inf \left\{t \leq u \leq T: \hat{L}_{u}=0\right\}$. On $\left\{\hat{\tau}^{*} \geq \tau_{d}\right\}$, liquidation occurs at $\tau_{d}$ since $L_{\tau_{d}}=0$. 
On $\left\{\hat{\tau}^{*}<\tau_{d}\right\}, \hat{\tau}^{*}$ is optimal since when $u<\hat{\tau}^{*}, L_{u}=\mathbf{1}_{\left\{u<\tau_{d}\right\}} \hat{L}_{u}>0$ and $L_{\hat{\tau}^{*}}=0$. Incorporating the observation of $\tau_{d}$, the optimal stopping time is $\tau^{*}=\hat{\tau}^{*} \wedge \tau_{d}$.

Given default has not occurred by time $t$, if it is optimal to liquidate immediately at some $(t, \mathbf{x})$, then we have $\hat{L}(t, \mathbf{x})=0$ and $G(t, \mathbf{x}) \leq 0$, which can be verified by substituting $\hat{L}(t, \mathbf{x})=0$ into (3.18). Hence, as a useful rule of thumb, if $G(t, \mathbf{x})>0$ for some $(t, \mathbf{x}) \in[0, T] \times \mathbb{R}^{n}$, then it is optimal to wait at the current $(t, \mathbf{x})$.

Further, if the defaultable claim is always underpriced by the market, i.e. $C(t, \mathbf{x}) \leq \tilde{C}(t, \mathbf{x})$ $\forall(t, \mathbf{x})$, then the holder is no better off than delaying liquidation till $T$. In this case, we observe that $J_{t}=0$ (see (2.6)) and $L_{t}=\mathbf{1}_{\left\{t<\tau_{d}\right\}}\left(\tilde{C}\left(t, \mathbf{X}_{t}\right)-C\left(t, \mathbf{X}_{t}\right)\right)$. Interestingly, the reverse is also true. Indeed, the optimality of $T$ as the liquidation time yields the inequality for the defaultable claim prices: $P_{t} \leq V_{t}=\tilde{P}_{t}$ a.s. for $t \leq T$, so the pre-default prices satisfy $\tilde{C}(t, \mathbf{x}) \geq C(t, \mathbf{x}) \forall(t, \mathbf{x})$.

\section{Applications to Defaultable Securities}

We proceed to illustrate our analysis for a number of defaultable securities, with an emphasis on how risk premia discrepancy affects the optimal liquidation strategies.

\subsection{Defaultable Bonds with Zero Recovery}

Consider a defaultable zero-coupon zero-recovery bond with face value 1 and maturity $T$. By a change of filtration $[6, \S 5.1 .1]$, the market price of the zero-coupon zero-recovery bond is given by

$$
P_{t}^{0}:=\mathbb{E}^{\mathbb{Q}}\left\{e^{-\int_{t}^{T} r_{v} d v} \mathbf{1}_{\left\{\tau_{d}>T\right\}} \mid \mathcal{G}_{t}\right\}=\mathbf{1}_{\left\{t<\tau_{d}\right\}} \mathbb{E}^{\mathbb{Q}}\left\{e^{-\int_{t}^{T}\left(r_{v}+\lambda_{v}\right) d v} \mid \mathcal{F}_{t}\right\}=\mathbf{1}_{\left\{t<\tau_{d}\right\}} C^{0}\left(t, \mathbf{X}_{t}\right),
$$

where $C^{0}$ denotes the market pre-default price that solves (3.13). Under the general Markovian credit risk model in Section 3.1, we can apply Theorem 3.4 with the quadruple $\left(1,0,0, \tau_{d}\right)$ to obtain the corresponding drift function.

Under the OU dynamics in Section 3.1, the pre-default price function $C^{0}(t, r, \lambda)$ is given explicitly by $[28, \S 7.1 .1]$ :

$$
C^{0}(t, r, \lambda)=e^{A(T-t)-B(T-t) r-D(T-t) \lambda},
$$

where

$$
\begin{aligned}
& B(s)=\frac{1-e^{-\kappa_{r} s}}{\kappa_{r}}, \quad D(s)=\frac{1-e^{-\kappa_{\lambda} s}}{\kappa_{\lambda}}, \\
& A(s)=\int_{0}^{s}\left[\frac{1}{2} \sigma_{r}^{2} B^{2}(z)+\rho \mu \sigma_{r} \sigma_{\lambda} B(z) D(z)+\frac{1}{2} \mu^{2} \sigma_{\lambda}^{2} D^{2}(z)-\kappa_{r} \theta_{r} B(z)-\mu \kappa_{\lambda} \theta_{\lambda} D(z)\right] d z .
\end{aligned}
$$

As a result, the drift function $G^{0}(t, r, \lambda)$ admits a separable form:

$$
\begin{aligned}
G^{0}(t, r, \lambda) & =C^{0}(t, r, \lambda)\left(B(T-t)\left(\tilde{\kappa}_{r}-\kappa_{r}\right) r+B(T-t)\left(\kappa_{r} \theta_{r}-\tilde{\kappa}_{r} \tilde{\theta}_{r}\right)\right. \\
& \left.+\left[D(T-t)\left(\tilde{\kappa}_{\lambda}-\kappa_{\lambda}\right)-\left(\frac{\tilde{\mu}}{\mu}-1\right)\right] \lambda+\mu D(T-t)\left(\kappa_{\lambda} \theta_{\lambda}-\tilde{\kappa}_{\lambda} \tilde{\theta}_{\lambda}\right)\right) .
\end{aligned}
$$

We can draw several insights on the liquidation timing from this drift function. If the market and the investor agree on the specifications of interest rate, i.e. $\kappa_{r}=\tilde{\kappa}_{r}$, and $\theta_{r}=\tilde{\theta}_{r}$, then $G^{0}(t, r, \lambda) / C^{0}(t, r, \lambda)$ is in fact linear in $\lambda$. Furthermore, if the slope $D(T-t)\left(\tilde{\kappa}_{\lambda}-\kappa_{\lambda}\right)-\left(\frac{\tilde{\mu}}{\mu}-1\right)$ and intercept $\mu D(T-t)\left(\kappa_{\lambda} \theta_{\lambda}-\tilde{\kappa}_{\lambda} \tilde{\theta}_{\lambda}\right)$ are of the same sign, then the optimal liquidation strategy 
must be trivial in view of Theorem 3.4. In contrast, if the slope and intercept differ in signs, the optimal stopping problem may be nontrivial and the sign of the slope determines qualitative properties of optimal stopping rules. For instance, suppose the slope is positive. We infer that it is optimal for the holder to wait at high default intensity where the corresponding $G^{0}$ and thus delayed liquidation premium are positive. The converse holds if the slope is negative.

If the investor disagrees with market only on event risk premium, i.e. $\mu \neq \tilde{\mu}$, then the drift function is reduced to $G^{0}(t, r, \lambda)=-C^{0}(t, r, \lambda)\left(\frac{\tilde{\mu}}{\mu}-1\right) \lambda$, which is of constant sign. This implies trivial strategies. If $\mu>\tilde{\mu}$, then $G^{0}>0$ and it is optimal to delay the liquidation until maturity. On the other hand, if $\mu<\tilde{\mu}$, then it is optimal to sell immediately. More general specifications of the event risk premium could depend on the state vector and may lead to nontrivial optimal stopping rules. Disagreement on mean level $\theta_{\lambda}$ has a similar effect to that of $\mu$.

If the investor disagrees with market only on speed of mean reversion, i.e. $\kappa_{\lambda} \neq \tilde{\kappa}_{\lambda}$, then $G^{0}(t, r, \lambda)=C^{0}(t, r, \lambda) D(T-t)\left[\left(\tilde{\kappa}_{\lambda}-\kappa_{\lambda}\right) \lambda+\mu \theta_{\lambda}\left(\kappa_{\lambda}-\tilde{\kappa}_{\lambda}\right)\right]$ with $D(T-t)>0$ before $T$, where the slope and intercept differ in signs. If $\kappa_{\lambda}<\tilde{\kappa}_{\lambda}$, the slope $\tilde{\kappa}_{\lambda}-\kappa_{\lambda}$ is positive and it is optimal to sell immediately at a low intensity, and thus, a high bond price. The converse holds for $\kappa_{\lambda}>\tilde{\kappa}_{\lambda}$.

We consider a numerical example where the interest rate is constant and the market default intensity $\lambda$ is chosen as the state vector $\mathbf{X}$ with OU dynamics. We employ the standard implicit PSOR algorithm to solve $\hat{L}(t, \lambda)$ through its variational inequality (3.18) over a uniform finite grid with von Neumann condition applied on the intensity boundary. The market parameters are $T=1, \mu=2, \kappa_{\lambda}=0.2, \theta_{\lambda}=0.015, r=0.03$, and $\sigma=0.02$, which are based on the estimates in $[9,10]$.

From formula (4.2), we observe a one-to-one correspondence between the market pre-default bond price $C^{0}$ and its default intensity $\lambda$ for any fixed $(t, r)$, namely,

$$
\lambda=\frac{-\log \left(C^{0}\right)+A(T-t)-B(T-t) r}{D(T-t)} .
$$

Substituting (4.5) into (3.19) and (3.20), we can characterize the sell region and delay region in terms of the observable pre-default market price $C^{0}$.

In the left panel of Figure 1, we assume that the investor agrees with the market on all parameters, but has a higher speed of mean reversion $\tilde{\kappa}_{\lambda}>\kappa_{\lambda}$. In this case, the investor tends to sell the bond at a high market price, which is consistent with our previous analysis in terms of drift function. If the bond price starts below 0.958 at time 0 , the optimal liquidation strategy for the investor is to hold and sell the bond as soon as the price hits the optimal boundary. If the bond price starts above 0.958 at time 0 , the optimal liquidation strategy is to sell immediately. In the opposite case where $\tilde{\kappa}_{\lambda}<\kappa_{\lambda}$ (see Figure 1(right)), the optimal liquidation strategy is reversed - it is optimal to sell at a lower boundary. In each cases, the sell region must lie within where $G$ is non-positive, and the straight line defined by $G=0$ can be viewed as a linear approximation of the optimal liquidation boundary.

Under the CIR dynamics in Section 3.1, $C^{0}$ admits closed-form formula [28, $\left.\S 7.2\right]$

$$
C^{0}(t, \mathbf{x})=\prod_{i=1}^{n} \mathbb{E}^{\mathbb{Q}}\left\{e^{-\int_{t}^{T}\left(w_{i}^{r}+\mu w_{i}^{\lambda}\right) X_{v}^{i} d v} \mid \mathbf{X}_{t}=\mathbf{x}\right\}=\prod_{i=1}^{n} A_{i}(T-t) e^{-B_{i}(T-t) x_{i}}
$$


where

$$
\begin{aligned}
& A_{i}(s)=\left[\frac{2 \Xi_{i} e^{\left(\Xi_{i}+\kappa_{i}\right) s / 2}}{\left(\Xi_{i}+\kappa_{i}\right)\left(e^{\Xi_{i} s}-1\right)+2 \Xi_{i}}\right]^{2 \kappa_{i} \theta_{i} / \sigma_{i}^{2}}, \\
& B_{i}(s)=\frac{2\left(e^{\Xi_{i} s}-1\right)\left(w_{i}^{r}+\mu w_{i}^{\lambda}\right)}{\left(\Xi_{i}+\kappa_{i}\right)\left(e^{\Xi_{i} s}-1\right)+2 \Xi_{i}}, \quad \text { and } \quad \Xi_{i}=\sqrt{\kappa_{i}^{2}+2 \sigma_{i}^{2}\left(w_{i}^{r}+\mu w_{i}^{\lambda}\right)} .
\end{aligned}
$$
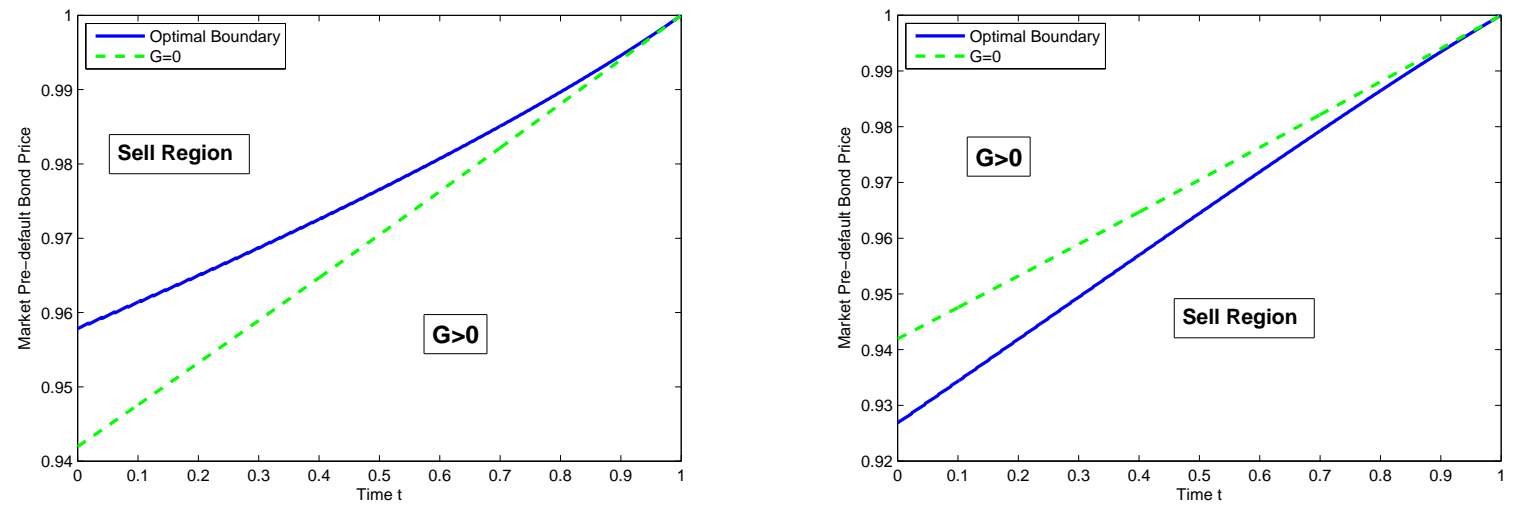

Figure 1: Optimal liquidation boundary in terms of market pre-default bond price under OU dynamics. We take $T=1, r=0.03, \sigma=0.02, \mu=\tilde{\mu}=2$, and $\theta_{\lambda}=\tilde{\theta}_{\lambda}=0.015$. Left panel: When $\kappa_{\lambda}=0.2<0.3=\tilde{\kappa}_{\lambda}$, the optimal boundary increases from 0.958 to 1 over time. Right panel: When $\kappa_{\lambda}=0.3>0.2=\tilde{\kappa}_{\lambda}$, the optimal boundary increases from 0.927 to 1 over time. The dashed straight line is defined by $G=0$, and we have $G \leq 0$ in both sell regions.
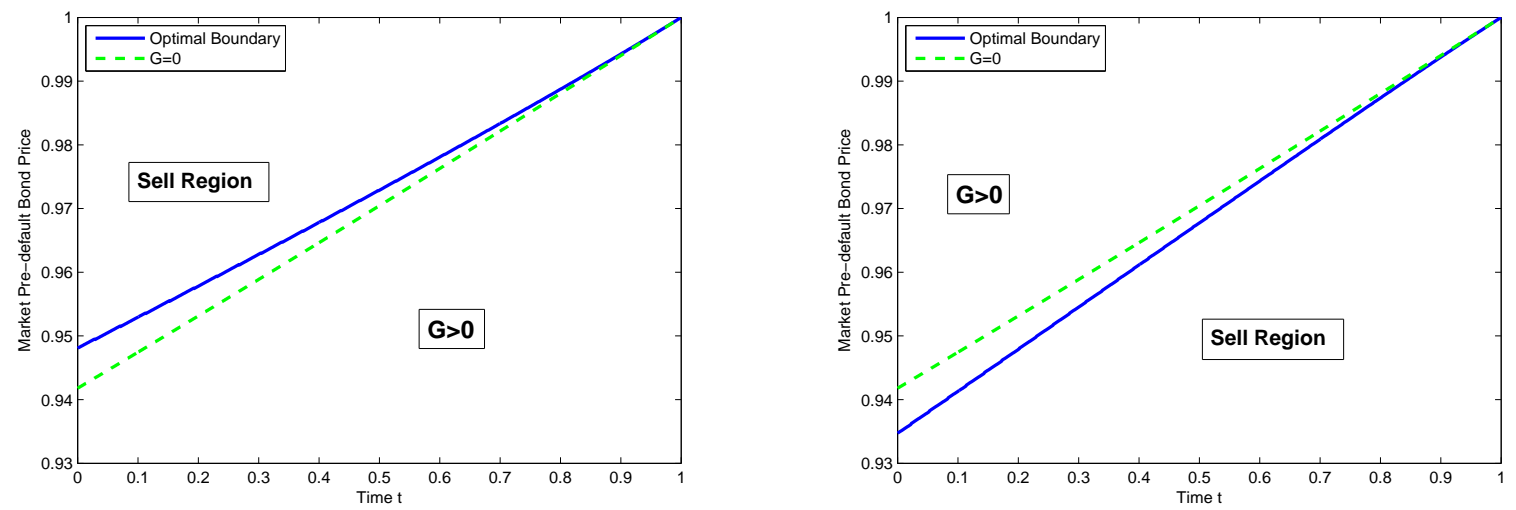

Figure 2: Optimal liquidation boundary in terms of market pre-default bond price under CIR dynamics. We take $T=1, r=0.03, \sigma=0.07, \mu=\tilde{\mu}=2$, and $\theta_{\lambda}=\tilde{\theta}_{\lambda}=0.015$. Left panel: When $\kappa_{\lambda}=0.2<0.3=\tilde{\kappa}_{\lambda}$, the optimal boundary increases from 0.948 to 1 over time. Right panel: When $\kappa_{\lambda}=0.3>0.2=\tilde{\kappa}_{\lambda}$, the optimal boundary increases from 0.935 to 1 over time. 
As a result, the drift function is given by

$$
G^{0}(t, \mathbf{x})=\left[\sum_{i=1}^{n}\left(\left[B_{i}(T-t)\left(\tilde{\kappa}_{i}-\kappa_{i}\right)-(\tilde{\mu}-\mu) w_{i}^{\lambda}\right] x_{i}+B_{i}(T-t)\left(\kappa_{i} \theta_{i}-\tilde{\kappa}_{i} \tilde{\theta}_{i}\right)\right)\right] C^{0}(t, \mathbf{x}),
$$

which is again linear in terms of $C^{0}(t, \mathbf{x})$.

To illustrate the optimal liquidation strategy, we consider a numerical example where interest rate is constant, $\mathbf{X}=\lambda$, and $\mathbf{w}^{\lambda}=\frac{1}{\mu}$. The benchmark specifications for the market default intensity $\lambda$ in the CIR dynamics are $T=1, \mu=2, \kappa_{\lambda}=0.2, \theta_{\lambda}=0.015, r=0.03$, and $\sigma=0.07$ based on the estimates from $[9,10]$. Like in the OU model, we can again express the sell region and delay region in terms of the pre-default market price $C^{0}$; see Figure 2.

\subsection{Recovery of Treasury and Market Value}

Extending the preceding analysis on defaultable bonds, we incorporate two principle ways of modeling recovery: the recovery of treasury or market value.

By the recovery of treasury, we assume that a recovery of $c$ times the value of the equivalent default-free bond is paid upon default. Therefore, the market pre-default bond price function is

$$
C^{R T}(t, \mathbf{x})=(1-c) C^{0}(t, \mathbf{x})+c \beta(t, \mathbf{x}),
$$

where $\beta(t, \mathbf{x}):=\mathbb{E}^{\mathbb{Q}}\left\{e^{-\int_{t}^{T} r_{v} d v} \mid \mathbf{X}_{t}=\mathbf{x}\right\}$ is the equivalent default-free bond price. Then, applying Theorem 3.4 with the quadruple $\left(1,0, c \beta, \tau_{d}\right)$, we obtain the corresponding drift function:

$$
G^{R T}(t, \mathbf{x})=-\left(\nabla_{x} C^{R T}(t, \mathbf{x})\right)^{T} \Sigma(t, \mathbf{x}) \boldsymbol{\phi}^{\tilde{\mathbb{Q}}, \mathbb{Q}}(t, \mathbf{x})+(c-1)(\tilde{\mu}(t, \mathbf{x})-\mu(t, \mathbf{x})) \hat{\lambda}(t, \mathbf{x}) C^{0}(t, \mathbf{x}) .
$$

If $c=0$, then $C^{R T}(t, \mathbf{x})=C^{0}(t, \mathbf{x})$ and $G^{R T}$ in (4.10) reduces to the drift function of the zerorecovery bond. If $c=1$, then $C^{R T}(t, \mathbf{x})=\beta(t, \mathbf{x})$ is the market price of a default-free bond, and risk premium discrepancy may arise only from the interest rate dynamics.

Here are two examples where the drift function $G^{R T}$ in (4.10) can be computed explicitly.

Example 4.1. Under OU model, $C^{R T}(t, r, \lambda)$ is computed according to $(4.9)$ with $C^{0}(t, r, \lambda)$ in (4.2) and $\beta(t, r, \lambda)=e^{\bar{A}(T-t)-B(T-t) r}$, where $\bar{A}(s)=\int_{0}^{s}\left[\frac{1}{2} \sigma_{r}^{2} B^{2}(z)-\kappa_{r} \theta_{r} B(z)\right] d z$ and $B(s)$ is defined in (4.3).

Example 4.2. Under the multi-factor CIR model, $C^{R T}(t, \mathbf{x})$ is found again from (4.9), where $C^{0}(t, \mathbf{x})$ is given in (4.6), and $\beta(t, \mathbf{x})$ is computed from (4.6) with $\mathbf{w}^{\lambda}=\mathbf{0}$ in (4.7) and (4.8).

As for the recovery of market value, we assume that at default the recovery is $c$ times the pre-default value $C_{\tau_{d^{-}}}^{R M V}$. The market pre-default price is given by

$$
C^{R M V}\left(t, \mathbf{X}_{t}\right)=\mathbb{E}^{\mathbb{Q}}\left\{e^{-\int_{t}^{T}\left(r_{v}+(1-c) \lambda_{v}\right) d v} \mid \mathcal{F}_{t}\right\}, \quad 0 \leq t \leq T .
$$

The corresponding drift function can be obtained by applying the quadruple $\left(1,0, c C^{R M V}, \tau_{d}\right)$ to Theorem 3.4.

Example 4.3. Under the OU model in Section 3.1, the price function $C^{R M V}(t, r, \lambda)$ is given by

$$
C^{R M V}(t, r, \lambda)=e^{\hat{A}(T-t)-B(T-t) r-\hat{D}(T-t) \lambda},
$$


where $B(s)$ is defined in (4.3),

$$
\begin{aligned}
& \hat{D}(s)=\frac{(1-c)\left(1-e^{-\kappa_{\lambda} s}\right)}{\kappa_{\lambda}}, \text { and } \\
& \hat{A}(s)=\int_{0}^{s}\left[\frac{1}{2} \sigma_{r}^{2} B^{2}(z)+\rho \mu \sigma_{r} \sigma_{\lambda} B(z) \hat{D}(z)+\frac{1}{2} \mu^{2} \sigma_{\lambda}^{2} \hat{D}^{2}(z)-\kappa_{r} \theta_{r} B(z)-\mu \kappa_{\lambda} \theta_{\lambda} \hat{D}(z)\right] d z .
\end{aligned}
$$

Example 4.4. Under the multi-factor CIR model, $C^{R M V}(t, \mathbf{x})$ admits the same formula as (4.6) but with $\boldsymbol{w}^{\lambda}$ replaced by $(1-c) \boldsymbol{w}^{\lambda}$ in (4.7) and (4.8).

\subsection{Optimal Liquidation of CDS}

In this section we consider liquidating a digital CDS position. The protection buyer pays a premium to the protection seller from time 0 until default or maturity $T$, whichever is first, at a fixed rate $p$ specified at contract inception. In return, she receives the payoff 1 if default occurs at or before $T$. The liquidation of the CDS position at time $t$ can be achieved by entering a CDS contract as a protection seller with the same credit reference and same maturity $T$ at the prevailing market spread $p_{t}^{m}$. After the liquidation, she receives the premium at rate $p_{t}^{m}$ and pays the premium at rate $p$ until default or the maturity $T$. If default occurs, the default payments from both CDS contracts will cancel. Hence the mark-to-market value of the CDS is given by

$$
\begin{aligned}
\mathbb{E}^{\mathbb{Q}}\left\{\int_{t}^{T} e^{-\int_{t}^{u} r_{v} d v}\left(p_{t}^{m}-p\right) \mathbf{1}_{\left\{u<\tau_{d}\right\}} d u \mid \mathcal{G}_{t}\right\} \\
=\mathbb{E}^{\mathbb{Q}}\left\{e^{-\int_{t}^{\tau_{t}} r_{v} d v} \mathbf{1}_{\left\{t<\tau_{d} \leq T\right\}} \mid \mathcal{G}_{t}\right\}-\mathbb{E}^{\mathbb{Q}}\left\{\int_{t}^{T} e^{-\int_{t}^{u} r_{v} d v} p \mathbf{1}_{\left\{u<\tau_{d}\right\}} d u \mid \mathcal{G}_{t}\right\} \\
=\mathbf{1}_{\left\{t<\tau_{d}\right\}} \mathbb{E}^{\mathbb{Q}}\left\{\int_{t}^{T} e^{-\int_{t}^{u}\left(r_{v}+\lambda_{v}\right) d v}\left(\lambda_{u}-p\right) d u \mid \mathcal{F}_{t}\right\}=: \mathbf{1}_{\left\{t<\tau_{d}\right\}} C^{C D S}\left(t, \mathbf{X}_{t}\right) .
\end{aligned}
$$

Here, the first equality follows from the fact that the market premium rate $p_{t}^{m}$ makes the values of two legs equal at time $t$.

Comparing (3.11) and (4.12), we apply the quadruple $\left(0,-p, 1, \tau_{d}\right)$ to Theorem 3.4 and obtain the drift function:

$$
G^{C D S}(t, \mathbf{x})=-\left(\nabla_{x} C^{C D S}(t, \mathbf{x})\right)^{T} \Sigma(t, \mathbf{x}) \boldsymbol{\phi}^{\tilde{\mathbb{Q}}, \mathbb{Q}}(t, \mathbf{x})+\left(1-C^{C D S}(t, \mathbf{x})\right)(\tilde{\mu}(t, \mathbf{x})-\mu(t, \mathbf{x})) \hat{\lambda}(t, \mathbf{x}) .
$$

If there is no discrepancy over mark-to-market risk premium, i.e. $\boldsymbol{\phi}^{\tilde{\mathbb{Q}}, \mathbb{Q}}(t, \mathbf{x})=\mathbf{0}$, then the sign of $G^{C D S}$ is determined by $\tilde{\mu}(t, \mathbf{x})-\mu(t, \mathbf{x})$ since $C^{C D S} \leq 1$. From this we infer that higher event risk premium (relative to market) implies delayed liquidation.

In general, the market pre-default value $C^{C D S}$ can be solved by PDE (3.13). If the state vector $\mathbf{X}$ admits $\mathrm{OU}$ or CIR dynamics, $C^{C D S}$, and thus $G^{C D S}$, is given in closed form, as illustrated in the following two examples.

Example 4.5. Under the OU dynamics, the pre-default value of CDS is given by the following integral:

$$
C^{C D S}(t, r, \lambda)=\int_{t}^{T} C^{0}(t, r, \lambda ; u)\left[\lambda e^{-\kappa_{\lambda}(u-t)}+\int_{t}^{u} e^{-\kappa_{r}(u-s)} g(s, u) d s-p\right] d u
$$


where $C^{0}(t, r, \lambda ; u)$ is given by $(4.2)$ with $T=u$ and

$$
g(s, u):=\mu \kappa_{\lambda} \theta_{\lambda}-\rho \mu \sigma_{r} \sigma_{\lambda} \frac{1-e^{-\kappa_{r}(u-s)}}{\kappa_{r}}-\left(\mu \sigma_{\lambda}\right)^{2} \frac{1-e^{-\kappa_{\lambda}(u-s)}}{\kappa_{\lambda}} .
$$

Example 4.6. Under the multi-factor CIR dynamics, the pre-default value of CDS is given by the following integral:

$$
C^{C D S}(t, \mathbf{x})=\int_{t}^{T} C^{0}(t, \mathbf{x} ; u)\left[\sum_{i=1}^{n}\left(\mu w_{i}^{\lambda}\left(\kappa_{i} \theta_{i} B_{i}(u-t)+B_{i}^{\prime}(u-t) x_{i}\right)\right)-p\right] d u,
$$

where $C^{0}(t, \mathbf{x} ; u)$ is given in (4.6) with $T=u$ and $B_{i}(s)$ in (4.8). See Chapter 7 of [28].

Example 4.7. For a forward CDS with start date $T_{a}<T$, the protection buyer pays premium at rate $p_{a}$ from $T_{a}$ until $\tau_{d}$ or maturity $T$, and receives 1 if $\tau_{d} \in\left[T_{a}, T\right]$. By direct computation, the pre-default market value is $C^{C D S}(t, \mathbf{x} ; T)-C^{C D S}\left(t, \mathbf{x} ; T_{a}\right), t<T_{a}$. Consequently, closed-form formulas for the drift function are available under OU or CIR dynamics by Examples 4.5 and 4.6.

We consider a numerical example where interest rate is constant and state vector $\mathbf{X}=\lambda$ follows the CIR dynamics. We assume that the investor agrees with the market on all parameters except the speed of mean reversion for default intensity. In the left panel of Figure 3 with $\kappa_{\lambda}=0.2<$ $0.3=\tilde{\kappa}_{\lambda}$, the optimal liquidation strategy is to sell as soon as the market CDS value reaches an upper boundary. In the case with $\kappa_{\lambda}=0.3>0.2=\tilde{\kappa}_{\lambda}$ (see Figure 3 (right)), the sell region is below the continuation region.
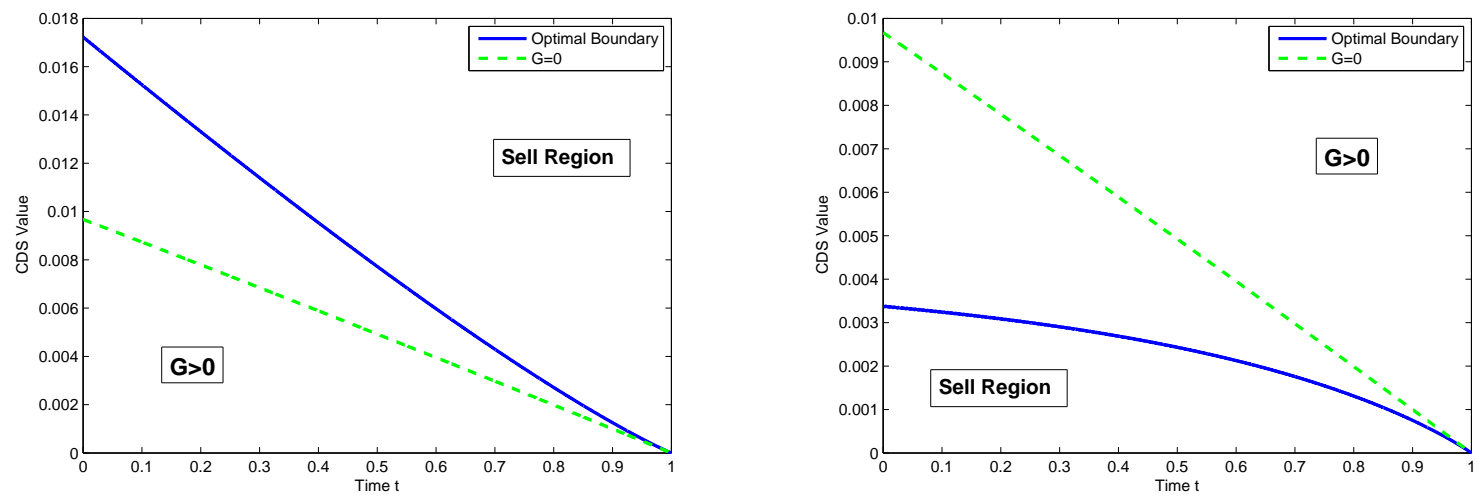

Figure 3: Optimal liquidation boundary in terms of market pre-default CDS value under CIR dynamics. We take $T=1, r=0.03, \sigma=0.07, p=0.02, \mu=\tilde{\mu}=2$, and $\theta_{\lambda}=\tilde{\theta}_{\lambda}=0.015$. Left panel: When $\kappa_{\lambda}=0.2<0.3=\tilde{\kappa}_{\lambda}$, liquidation occurs at an upper boundary that decreases from 0.0172 to 0 over $t \in[0,1]$. Right panel: When $\kappa_{\lambda}=0.3>0.2=\tilde{\kappa}_{\lambda}$, the CDS is liquidated at a lower liquidation boundary, which decreases from 0.00338 to 0 over time. In both cases, the dashed line defined by $G=0$ lies within the continuation region.

\section{Optimal Buying and Selling}

Next, we adapt our model to study the optimal buying and selling problem. Consider an investor whose objective is to maximize the revenue through a buy/sell transaction of a defaultable claim 
$\left(Y, A, R, \tau_{d}\right)$ with market price process $P$ in (2.4). The problem is studied separately under two scenarios, namely, when the short sale of the defaultable claim is permitted or prohibited.

If the investor seeks to purchase a defaultable claim from the market, the optimal purchase timing problem and the associated delayed purchase premium can be defined as:

$$
V_{t}^{b}=\underset{\tau^{b} \in \mathcal{T}_{t, T}}{\operatorname{essinf}} \mathbb{E}^{\tilde{\mathbb{Q}}}\left\{e^{-\int_{t}^{\tau^{b}} r_{v} d v} P_{\tau^{b}} \mid \mathcal{G}_{t}\right\}, \quad \text { and } \quad L_{t}^{b}:=P_{t}-V_{t}^{b} \geq 0 .
$$

\subsection{Optimal Timing with Short Sale Possibility}

When short sale is permitted, there is no restriction on the ordering of purchase time $\tau^{b}$ and sale time $\tau^{s}$. The investor's investment timing is found from the optimal double-stopping problem:

$$
\mathcal{U}_{t}:=\operatorname{ess}_{\tau^{b} \in \mathcal{T}_{t, T}, \tau^{s} \in \mathcal{T}_{t, T}} \mathbb{E}^{\tilde{\mathbb{Q}}}\left\{e^{-\int_{t}^{\tau^{s}} r_{v} d v} P_{\tau^{s}}-e^{-\int_{t}^{\tau^{b}} r_{v} d v} P_{\tau^{b}} \mid \mathcal{G}_{t}\right\}
$$

Since the defaultable claim will mature at $T$, we interpret the choice of $\tau^{b}=T$ or $\tau^{s}=T$ as no buy/sell transaction at $T$.

In fact, we can separate $\mathcal{U}$ into two optimal (single) stopping problems. Precisely, we have

$$
\begin{aligned}
\mathcal{U}_{t} & =\left(\underset{\tau^{s} \in \mathcal{T}_{t, T}}{\operatorname{essip} \sup } \mathbb{E}^{\tilde{\mathbb{Q}}}\left\{e^{-\int_{t}^{\tau^{s}} r_{v} d v} P_{\tau^{s}} \mid \mathcal{G}_{t}\right\}-P_{t}\right)+\left(P_{t}-\underset{\tau^{b} \in \mathcal{T}_{t, T}}{\operatorname{essinf}} \mathbb{E}^{\tilde{\mathbb{Q}}}\left\{e^{-\int_{t}^{\tau^{b}} r_{v} d v} P_{\tau^{b}} \mid \mathcal{G}_{t}\right\}\right) \\
& =L_{t}+L_{t}^{b}
\end{aligned}
$$

Hence, we have separated $\mathcal{U}$ into a sum of the delayed liquidation premium and the delayed purchase premium. As a result, the optimal sale time $\tau^{s *}$ does not depends on the choice of the optimal purchase time $\tau^{b *}$.

The timing decision again depends crucially on the sub/super-martingale properties of discounted market price under measure $\tilde{\mathbb{Q}}$. Under the Markovian market model in Section 3, we can apply Theorem 3.4 to describe the optimal purchase and sale strategies in terms of the drift function $G(t, \mathbf{x})$ in $(3.16)$.

Proposition 5.1. If $G(t, \mathbf{x}) \geq 0 \forall(t, \mathbf{x}) \in[0, T] \times \mathbb{R}^{n}$, then it is optimal to immediately buy the defaultable claim and hold it till maturity $T$, i.e. $\tau^{b *}=t$ and $\tau^{s *}=T$ are optimal for $\mathcal{U}_{t}$. If $G(t, \mathbf{x}) \leq 0 \forall(t, \mathbf{x}) \in[0, T] \times \mathbb{R}^{n}$, then it is optimal to immediately short sell the claim and maintain the position till $T$, i.e. $\tau^{s *}=t$ and $\tau^{b *}=T$ are optimal for $\mathcal{U}_{t}$.

\subsection{Sequential Buying and Selling}

Prohibiting the short sale of defaultable claims implies the ordering: $\tau^{b} \leq \tau^{s} \leq T$. Therefore, the investor's value function is given by

$$
U_{t}:=\operatorname{ess}_{\tau^{b} \in \mathcal{T}_{t, T}, \tau^{s} \in \mathcal{T}_{\tau^{b}, T}} \mathbb{E}^{\tilde{\mathbb{Q}}}\left\{e^{-\int_{t}^{\tau^{s}} r_{v} d v} P_{\tau^{s}}-e^{-\int_{t}^{\tau^{b}} r_{v} d v} P_{\tau^{b}} \mid \mathcal{G}_{t}\right\}
$$

The difference $\mathcal{U}_{t}-U_{t} \geq 0$ can be viewed as the cost of the short sale constraint to the investor. 
Lemma 5.2. The optimal double-stopping problem $U_{t}$ can be expressed as

$$
U_{t}=Z_{t}^{-1} \operatorname{esssup}_{\tau^{b} \in \mathcal{T}_{t, T}, \tau^{s} \in \mathcal{T}_{\tau^{b}, T}} \mathbb{E}^{\mathbb{Q}}\left\{\int_{\tau^{b}}^{\tau^{s}} e^{-\int_{t}^{u} r_{v} d v} d[P, Z]_{u} \mid \mathcal{G}_{t}\right\},
$$

where $Z_{t}=\mathbb{E}^{\mathbb{Q}}\left\{\frac{d \tilde{\mathbb{Q}}}{d \mathbb{Q}} \mid \mathcal{G}_{t}\right\}$.

Proof. This follows from the same argument for Lemma 2.2, with $t$ and $\tau$ in (2.12) replaced by $\tau^{b}$ and $\tau^{s}$ respectively.

Under the Markovian market in Section 3, we can apply Lemma 5.2 to deduce the optimal buy/sell strategy in the extreme cases analogues to Theorem 3.4.

Proposition 5.3. If $G(t, \mathbf{x}) \geq 0 \forall(t, \mathbf{x}) \in[0, T] \times \mathbb{R}^{n}$, then it is optimal to purchase the defaultable claim immediately and hold until maturity, i.e. $\tau^{b *}=t$ and $\tau^{s *}=T$ are optimal for $U_{t}$.

If $G(t, \mathbf{x}) \leq 0 \forall(t, \mathbf{x}) \in[0, T] \times \mathbb{R}^{n}$, then it is optimal to never purchase the claim, i.e. $\tau^{b *}=\tau^{s *}=T$ is optimal for $U_{t}$.

In fact, the optimal double-stopping problem $U_{t}$ can be expressed as a sequential optimal stopping problem. Once the purchase is made, the investor faces a pure liquidation problem as studied in Section 2.2 (see (2.7)). Therefore, we can first solve for the optimal sale strategy (described by a sale boundary), and use it as the input to solve for the optimal purchase timing. This observation also means that the investor's optimal sale boundary will be the same whether or not short sale is permitted. However, prior to the purchase, the investor can sell at the desired sale boundary only if there is no short sale constraint.

In Figure 4, we show a numerical example for a defaultable zero-coupon zero-recovery bond where interest rate is constant and $\lambda$ follows the CIR dynamics. The investor agrees with the market on all parameters except the speed of mean reversion for default intensity. When $\kappa_{\lambda}<\tilde{\kappa}_{\lambda}$, the optimal strategy is to buy as soon as the price enters the purchase region and subsequently sell at the (higher) optimal liquidation boundary. When $\kappa_{\lambda}>\tilde{\kappa}_{\lambda}$, the optimal liquidation boundary is below the purchase boundary. However, it is possible that the investor buys at a lower price and subsequently sells at a higher price since both boundaries are increasing. It is also possible to buy-high-sell-low, realizing a loss on these sample paths. On average, the optimal sequential buying and selling strategy enables the investor to profit from the price discrepancy. Finally, when short sale is allowed, the investor's strategy follows the corresponding boundaries without the buy-first/sell-later constraint.

\section{Extensions and Conclusions}

In this section, we discuss the extensions to a jump-diffusion default intensity model as well as a defaultable equity model.

\subsection{Jump-Diffusion Default Intensity}

Suppose the default intensity and interest rate are driven by a state vector $\mathbf{X}^{\prime}$ with the affine jump-diffusion dynamics:

$$
d \mathbf{X}_{t}^{\prime}=b\left(t, \mathbf{X}_{t}^{\prime}\right) d t+\Sigma\left(t, \mathbf{X}_{t}^{\prime}\right) d \mathbf{W}_{t}^{\mathbb{Q}}+d \mathbf{J}_{t}
$$



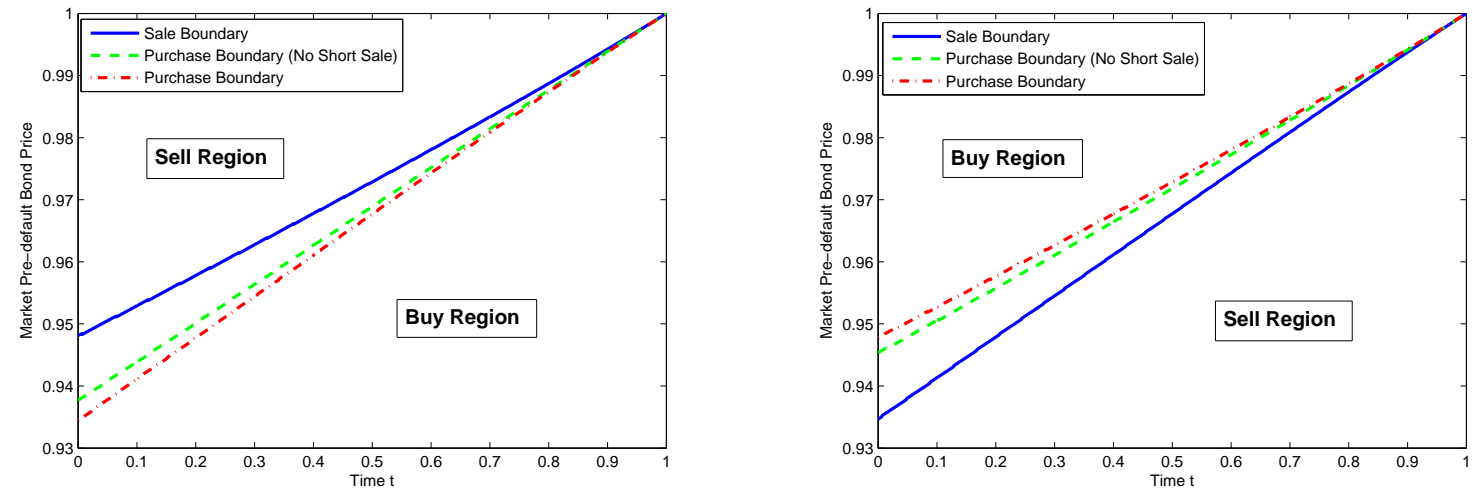

Figure 4: Optimal purchase and liquidation boundaries in the CIR model. The common parameters are $T=1, r=0.03, \sigma=0.07, \mu=\tilde{\mu}=2$, and $\theta_{\lambda}=\tilde{\theta}_{\lambda}=0.015$. Left panel: When $\kappa_{\lambda}=0.2<0.3=\tilde{\kappa}_{\lambda}$, the short sale constraint moves the purchase boundary higher. Both purchase boundaries, with or without short sale, are dominated by the liquidation boundary. Right panel: When $\kappa_{\lambda}=0.3>0.2=\tilde{\kappa}_{\lambda}$, the short sale constraint moves the purchase boundary lower. The liquidation boundary lies below both purchase boundaries.

where $\mathbf{J}$ is a $n$-dimensional pure jump process taking values in $\mathbb{R}^{n}$. Under $\mathbb{Q}$, the Markovian jump intensity of $\mathbf{J}$ is $l\left(t, \mathbf{X}_{t}^{\prime}\right)$ and its jump sizes $\mathbf{Y}_{1}, \mathbf{Y}_{2}, \ldots$ are independent and identically distributed with a common probability density function $f(\mathbf{y})$. Let $T_{i}$ be the $i$ th jump time of $\mathbf{J}$ and $N_{t}^{J}=\sum_{i \geq 1} \mathbf{1}_{\left\{T_{i} \leq t\right\}}$ be the the counting process associated with $\mathbf{J}$. Under the investor's measure $\tilde{\mathbb{Q}}$, we replace $b$ with $\tilde{b}, \mathbf{W}^{\mathbb{Q}}$ with $\mathbf{W}^{\tilde{\mathbb{Q}}}$, and denote the $\tilde{\mathbb{Q}}$-intensity of $\mathbf{J}$ by $\tilde{l}\left(t, \mathbf{X}_{t}^{\prime}\right)$ and jump size density by $\tilde{f}(\mathbf{y})$.

The two pricing measures $\mathbb{Q}$ and $\tilde{\mathbb{Q}}$ are related by the Radon-Nikodym derivative:

$$
\frac{d \tilde{\mathbb{Q}}}{d \mathbb{Q}} \mid \mathcal{G}_{t}=\mathcal{E}\left(-\phi^{\tilde{\mathbb{Q}}, \mathbb{Q}} \cdot \mathbf{W}^{\mathbb{Q}}\right)_{t} \mathcal{E}\left(\frac{\tilde{\mu}}{\mu} M^{\mathbb{Q}}\right)_{t} K_{t}
$$

where the first two terms are given in (3.4) and (3.5) respectively, while $K$ is defined by

$$
K_{t}:=\exp \left(\int_{0}^{t} \int_{\mathbb{R}^{n}}\left(l\left(v, \mathbf{X}_{v}^{\prime}\right) f(\mathbf{y})-\tilde{l}\left(v, \mathbf{X}_{v}^{\prime}\right) \tilde{f}(\mathbf{y})\right) d \mathbf{y} d v\right) \prod_{i=1}^{N_{t}^{J}} \frac{\tilde{l}\left(T_{i}, \mathbf{X}_{T_{i}}^{\prime}\right) \tilde{f}\left(\mathbf{Y}_{i}\right)}{l\left(T_{i}, \mathbf{X}_{T_{i}}^{\prime}\right) f\left(\mathbf{Y}_{i}\right)}
$$

Consequently, on top of the mark-to-market risk and event risk premia, the investor can potentially disagree with the market over jump intensity and jump size distribution of $\mathbf{X}^{\prime}$, allowing for a richer structure of price discrepancy as well as the optimal liquidation strategy.

As in Theorem 3.4, we compute the drift function in terms of pre-default price $C$ and default risk premia, namely,

$$
\begin{aligned}
G^{J}(t, \mathbf{x}) & =-\left(\nabla_{x} C(t, \mathbf{x})\right)^{T} \Sigma(t, \mathbf{x}) \boldsymbol{\phi}^{\tilde{\mathbb{Q}}, \mathbb{Q}}(t, \mathbf{x})+(R(t, \mathbf{x})-C(t, \mathbf{x}))(\tilde{\mu}(t, \mathbf{x})-\mu(t, \mathbf{x})) \hat{\lambda}(t, \mathbf{x}) \\
& +\int_{\mathbb{R}^{n}}(C(t, \mathbf{x}+\mathbf{y})-C(t, \mathbf{x}))(\tilde{l}(t, \mathbf{x}) \tilde{f}(\mathbf{y})-l(t, \mathbf{x}) f(\mathbf{y})) d \mathbf{y}
\end{aligned}
$$

which has a third term arising from $\mathbf{J}$. We observe that the first two components of $G^{J}$ share the same functional form as $G$ in (3.16), though the price function $C$ is derived from the jump-diffusion 
model. Even if the investor and the market assign the same mark-to-market risk and event risk premia, discrepancy over jump intensity and distribution will yield different liquidation strategies. Under quite general affine jump-diffusion models, Duffie et al. [11] provide an analytical treatment of transform analysis, which can be used for the computation of our drift function.

\subsection{Options on a Defaultable Stock}

Another useful application is the liquidation of options written on a defaultable equity. Under the market measure $\mathbb{Q}$, the defaultable stock price $S$ evolves according to

$$
d S_{t}=\left(r_{t}+\lambda_{t}\right) S_{t} d t+\eta S_{t} d B_{t}^{\mathbb{Q}}-S_{t-} d N_{t},
$$

where $N_{t}=\mathbf{1}_{\left\{t \geq \tau_{d}\right\}}$ is the default counting process, $B^{\mathbb{Q}}$ is a standard $\mathbb{Q}$-Brownian motion independent of $E$ and $\mathbf{W}^{\mathbb{Q}}$, and $r_{t}=r\left(t, \mathbf{X}_{t}\right)$ is driven by $\mathbf{X}$ in (3.2). Like in the Markovian credit risk model in Section 3 , we denote under $\mathbb{Q}($ resp. $\tilde{\mathbb{Q}})$ the default intensity by $\lambda\left(t, \mathbf{X}_{t}\right)\left(\operatorname{resp} . \tilde{\lambda}\left(t, \mathbf{X}_{t}\right)\right)$, default risk premia by $\boldsymbol{\phi}^{\mathbb{Q}, \mathbb{P}}\left(t, \mathbf{X}_{t}\right)$ and $\mu\left(t, \mathbf{X}_{t}\right)\left(\operatorname{resp} . \phi^{\tilde{\mathbb{Q}}, \mathbb{P}}\left(t, \mathbf{X}_{t}\right)\right.$ and $\left.\tilde{\mu}\left(t, \mathbf{X}_{t}\right)\right)$. Denote by $\mathbb{G}$ the full filtration generated by $\mathbf{X}$ and $S$. For similar defaultable equity models, we refer to $[3,25]$, among others.

The two pricing measures $\mathbb{Q}$ and $\tilde{\mathbb{Q}}$ are related by the Radon-Nikodym derivative:

$$
\bar{Z}_{t}=\frac{d \tilde{\mathbb{Q}}}{d \mathbb{Q}} \mid \mathcal{G}_{t}=\mathcal{E}\left(-\phi^{\tilde{\mathbb{Q}}, \mathbb{Q}} \cdot \mathbf{W}^{\mathbb{Q}}\right)_{t} \mathcal{E}\left(\frac{\tilde{\mu}}{\mu} M^{\mathbb{Q}}\right)_{t} \mathcal{E}\left(-\gamma^{\tilde{\mathbb{Q}}, \mathbb{Q}} B^{\mathbb{Q}}\right)_{t}
$$

where the first two terms are given in (3.4) and (3.5), while the third term is defined by

$$
\mathcal{E}\left(-\gamma^{\tilde{\mathbb{Q}}, \mathbb{Q}} B^{\mathbb{Q}}\right)_{t}:=\exp \left(-\frac{1}{2} \int_{0}^{t}\left(\gamma_{u}^{\tilde{\mathbb{Q}}, \mathbb{Q}}\right)^{2} d u-\int_{0}^{t} \gamma_{u}^{\tilde{\mathbb{Q}}, \mathbb{Q}} d B_{u}^{\mathbb{Q}}\right)
$$

where $\gamma^{\tilde{\mathbb{Q}}, \mathbb{Q}}$ is the risk premium for the Brownian motion $B$ driving $S$. It is related to the event risk premia via the equation:

$$
\gamma^{\tilde{\mathbb{Q}}, \mathbb{Q}}\left(t, \mathbf{X}_{t}\right)=\frac{\left(\mu\left(t, \mathbf{X}_{t}\right)-\tilde{\mu}\left(t, \mathbf{X}_{t}\right)\right) \hat{\lambda}\left(t, \mathbf{X}_{t}\right)}{\eta}
$$

by the martingale property of the discounted stock price under both $\mathbb{Q}$ and $\tilde{\mathbb{Q}}$.

Denote the corresponding market price function of option $F$ by

$$
P(t, \mathbf{x}, s)=\mathbb{E}^{\mathbb{Q}}\left\{e^{-\int_{t}^{T} r_{v} d v} F\left(S_{T}\right) \mid \mathbf{X}_{t}=\mathbf{x}, S_{t}=s\right\} .
$$

The option holder faces the optimal liquidation problem:

$$
\bar{V}(t, \mathbf{x}, s):=\operatorname{esssup}_{\tau \in \mathcal{T}_{t, T}} \mathbb{E}^{\tilde{\mathbb{Q}}}\left\{e^{-\int_{t}^{\tau} r_{v} d v} P\left(\tau, \mathbf{X}_{\tau}, S_{\tau}\right) \mid \mathbf{X}_{t}=\mathbf{x}, S_{t}=s\right\} .
$$

In the event $\left\{\tau<\tau_{d}\right\}$, the option is sold to the market. Otherwise, the value of the holder's position $^{1}$ is $P\left(\tau, \mathbf{X}_{\tau}, 0\right)=F(0) \mathbb{E}^{\mathbb{Q}}\left\{e^{-\int_{\tau}^{T} r_{v} d v} \mid \mathbf{X}_{\tau}\right\}, \tau \geq \tau_{d}$. The delayed liquidation premium, defined by $\bar{L}:=\bar{V}-P$, can be analyzed through a probabilistic representation.

\footnotetext{
${ }^{1}$ The holder can lock in this value by shorting $F(0)$ units of the default-free zero-coupon bond with maturity $T$ at $\tau \geq \tau_{d}$, yielding zero payoff at $T$.
} 
Proposition 6.1. Under the Markovian credit risk model, the delayed liquidation premium for option $F$ at time $t \leq \tau_{d}$ is given by

$$
\bar{L}(t, \mathbf{x}, s)=\underset{\tau \in \mathcal{T}_{t, T}}{\operatorname{ess} \sup } \mathbb{E}^{\tilde{\mathbb{Q}}}\left\{\int_{t}^{\tau} e^{-\int_{t}^{u} r_{v} d v} \bar{G}\left(u, \mathbf{X}_{u}, S_{u}\right) d u \mid \mathbf{X}_{t}=\mathbf{x}, S_{t}=s\right\}
$$

where $\bar{G}:[0, T] \times \mathbb{R}^{n} \times[0, \infty) \mapsto \mathbb{R}$ is defined by

$$
\begin{aligned}
\bar{G}(t, \mathbf{x}, s)= & -\left(\nabla_{x} P(t, \mathbf{x}, s)\right)^{T} \Sigma(t, \mathbf{x}) \boldsymbol{\phi}^{\tilde{\mathbb{Q}}, \mathbb{Q}}(t, \mathbf{x}) \\
& +\left(s \frac{\partial P}{\partial s}(t, \mathbf{x}, s)+P(t, \mathbf{x}, 0)-P(t, \mathbf{x}, s)\right)(\tilde{\mu}(t, \mathbf{x})-\mu(t, \mathbf{x})) \hat{\lambda}(t, \mathbf{x}) .
\end{aligned}
$$

If $\bar{G}(t, \mathbf{x}, s) \geq 0 \forall(t, \mathbf{x}, s)$, then it is optimal to delay the liquidation until maturity $T$. If $\bar{G}(t, \mathbf{x}, s) \leq 0 \forall(t, \mathbf{x}, s)$, then it is optimal to liquidate the option immediately.

Proof. In view of (6.4) and (6.5), the stochastic bracket between $P$ and $\bar{Z}$ is given by

$$
d P_{u} d \bar{Z}_{u}=\bar{Z}_{u-}\left[\bar{G}\left(u, \mathbf{X}_{u}, S_{u}\right) d u+\left(\frac{\tilde{\mu}_{u}}{\mu_{u}}-1\right)\left(P\left(u, \mathbf{X}_{u}, 0\right)-P\left(u, \mathbf{X}_{u}, S_{u-}\right)\right) d M_{u}^{\mathbb{Q}}\right],
$$

where $\bar{G}(t, \mathbf{x}, s)$ is defined in (6.8). Using argument similar for Lemma 2.2, we obtain

$$
\bar{L}_{t}=\bar{Z}_{t}^{-1} \underset{\tau \in \mathcal{T}_{t, T}}{\operatorname{ess} \sup } \mathbb{E}^{\mathbb{Q}}\left\{\int_{t}^{\tau} \bar{Z}_{u} e^{-\int_{t}^{u} r_{v} d v} \bar{G}\left(u, \mathbf{X}_{u}, S_{u}\right) d u \mid \mathcal{G}_{t}\right\}
$$

which yields (6.7) by a change of measure from $\mathbb{Q}$ to $\tilde{\mathbb{Q}}$.

In the drift function (6.8), the first component is related to the mark-to-market risk premium $\phi^{\tilde{\mathbb{Q}}, \mathbb{Q}}$, and the second part depends on the event risk premium and the convexity (or gamma) of the price $P(t, \mathbf{x}, s)$. Indeed, if the gamma $P_{s s}(t, \mathbf{x}, s) \geq 0$, then $s \frac{\partial P}{\partial s}(t, \mathbf{x}, s)+P(t, \mathbf{x}, 0)-P(t, \mathbf{x}, s) \geq 0$. If there is no discrepancy over the mark-to-market risk premium, then the optimal stopping rule is simplified to direct comparison of the event risk premia. In summary,

Corollary 6.2. Suppose that the option price function $s \mapsto P(t, \mathbf{x}, s)$ is convex and $\boldsymbol{\phi}^{\tilde{\mathbb{Q}}, \mathbb{Q}}(t, \mathbf{x})=\mathbf{0}$ for every $(t, \mathbf{x}) \in[0, T] \times \mathbb{R}^{n}$. If $\tilde{\mu}(t, \mathbf{x}) \geq \mu(t, \mathbf{x})$ (resp. $\left.\tilde{\mu}(t, \mathbf{x}) \leq \mu(t, \mathbf{x})\right) \forall(t, \mathbf{x})$, then $\tau^{*}=T$ (resp. $\tau^{*}=t$ ) is optimal for $\bar{L}_{t}$.

Corollary 6.2 can be applied to European call and put options. Interestingly, we observe the following Put-Call parity in terms of optimal liquidation timing.

Proposition 6.3. Suppose the interest rate is deterministic. The investor's optimal strategies for selling a European Call and for selling a European Put, with the same underlying S, strike and maturity, are identical.

Proof. Denote the interest rate function by $r(\cdot)$ and the call and put price functions by $P^{C a l l}(t, \mathbf{x}, s)$ and $P^{P u t}(t, \mathbf{x}, s)$, with terminal payoffs $\left(S_{T}-K\right)^{+}$and $\left(K-S_{T}\right)^{+}$respectively. Applying the Put-Call parity and that $\left(e^{-\int_{0}^{u} r(v) d v} S_{u}\right)_{u \geq 0}$ is a $(\tilde{\mathbb{Q}}, \mathbb{G})$-martingale, we obtain

$$
\begin{aligned}
\mathbb{E}^{\tilde{\mathbb{Q}}}\left\{e^{-\int_{0}^{\tau} r(v) d v} P^{C a l l}\left(\tau, \mathbf{X}_{\tau}, S_{\tau}\right)\right\} & =\mathbb{E}^{\tilde{\mathbb{Q}}}\left\{e^{-\int_{0}^{\tau} r(v) d v}\left(P^{P u t}\left(\tau, \mathbf{X}_{\tau}, S_{\tau}\right)+S_{\tau}-K e^{-\int_{\tau}^{T} r(v) d v}\right)\right\} \\
& =\mathbb{E}^{\tilde{\mathbb{Q}}}\left\{e^{-\int_{0}^{\tau} r(v) d v} P^{P u t}\left(\tau, \mathbf{X}_{\tau}, S_{\tau}\right)\right\}+S_{0}-K e^{-\int_{0}^{T} r(v) d v}
\end{aligned}
$$


Since the last two terms do not depend on the choice of $\tau$, it follows that

$$
\underset{\tau \in \mathcal{T}_{0, T}}{\arg \max } \mathbb{E}^{\tilde{\mathbb{Q}}}\left\{e^{-\int_{0}^{\tau} r(v) d v} P^{C a l l}\left(\tau, \mathbf{X}_{\tau}, S_{\tau}\right)\right\}=\underset{\tau \in \mathcal{T}_{0, T}}{\arg \max } \mathbb{E}^{\tilde{\mathbb{Q}}}\left\{e^{-\int_{0}^{\tau} r(v) d v} P^{P u t}\left(\tau, \mathbf{X}_{\tau}, S_{\tau}\right)\right\} .
$$

\subsection{Conclusions}

In summary, we have provided a flexible mathematical model for the optimal liquidation of various credit derivatives under price discrepancy. We have identified the situations where the optimal timing is trivial and also solved for the cases when sophisticated strategies are involved. The optimal liquidation framework enables investors to quantify their views on default risk, extract profit from price discrepancy, and perform more effective risk management. Our model can also be modified and extended to incorporate single or multiple buying and selling decisions.

For future research, a natural direction is to consider the investment timing problem under other default risk models. Also, liquidation problems are important for multi-name credit derivatives or a portfolio of derivatives in general. To this end, the structure of dependency between multiple risk factors is crucial in modeling price dynamics. Finally, efficient and robust calibration methods are challenging but also essential.

\section{References}

[1] R. F. Almgren. Optimal execution with nonlinear impact functions and trading-enhanced risk. Applied Mathematical Finance, 10:1-18, 2003.

[2] S. Azizpour, K. Giesecke, and B. Kim. Premia for correlated default risk. Journal of Economic Dynamics and Control, 35(8):1340-1357, 2011.

[3] E. Bayraktar. Pricing options on defaultable stocks. Applied Mathematical Finance, 15(3):277-304, 2008.

[4] A. Berndt, R. Douglas, D. Duffie, M. Ferguson, and D. Schranz. Measuring default risk premia from default swap rates and EDFs. Working Paper, Carnegie Mellon University., 2005.

[5] T. R. Bielecki, M. Jeanblanc, and M. Rutkowski. Pricing and trading credit default swaps in a hazard process model. Annals of Applied Probability, 18(6):2495-2529, 2008.

[6] T. R. Bielecki and M. Rutkowski. Credit Risk: Modeling, Valuation and Hedging. Springer Finance, 2002.

[7] R. Cont and A. Minca. Recovering portfolio default intensities implied by CDO quotes. Mathematical Finance, 2011. forthcoming.

[8] B. Cornell, J. Cvitanić, and L. Goukasian. Optimal investing with perceived mispricing. 2007. Working paper.

[9] J. Driessen. Is default event risk priced in corporate bonds? Review of Financial Studies, 18(1):165$195,2005$.

[10] G. R. Duffee. Estimating the price of default risk. Review of Financial Studies, 12(1):197-226, 1999.

[11] D. Duffie, J. Pan, and K. J. Singleton. Transform analysis and asset pricing for affine jump-diffusions. Econometrica, 68(6):1343-1376, 2000. 
[12] D. Duffie and K. J. Singleton. Modeling term structures of defaultable bonds. Review of Financial Studies, 12(4):687-720, 1999.

[13] E. Ekström, C. Lindberg, J. Tysk, and H. Wanntorp. Optimal liquidation of a call spread. Journal of Applied Probability, 47(2):586-593, 2010.

[14] H. Föllmer and M. Schweizer. Hedging of contingent claims under incomplete information. In M.H.A. Davis and R.J. Elliot, editors, Applied Stochastic Analysis, Stochastics Monographs, volume 5, pages 389 - 414. Gordon and Breach, London/New York, 1990.

[15] M. Frittelli. The minimal entropy martingale measure and the valuation problem in incomplete markets. Mathematical Finance, 10(1):39-52, 2000.

[16] T. Fujiwara and Y. Miyahara. The minimal entropy martingale measures for geometric Lévy processes. Finance Stoch., 7(4):509-531, 2003.

[17] D. Hobson. Stochastic volatility models, correlation, and the q-optimal measure. Mathematical Finance, 14(4):537-556, 2004.

[18] R. A. Jarrow, D. Lando, and F. Yu. Default risk and diversification: Theory and empirical implications. Mathematical Finance, 15(1):1-26, 2005.

[19] R. A. Jarrow and S. M. Turnbull. Pricing derivatives on financial securities subject to credit risk. Journal of Finance, 50(1):53-85, 1995.

[20] I. Karatzas and S. E. Shreve. Brownian Motion and Stochastic Calculus. Springer, New York, 1991.

[21] I. Karatzas and S. E. Shreve. Methods of Mathematical Finance. Springer, New York, 1998.

[22] D. Lando. On Cox processes and credit risky securities. Review of Derivatives Research, 2(2-3):99-120, 1998.

[23] T. Leung and M. Ludkovski. Accounting for risk aversion in derivatives purchase timing. September 2011. Submitted.

[24] T. Leung and M. Ludkovski. Optimal timing to purchase options. SIAM Journal on Financial Mathematics, 2011. forthcoming.

[25] V. Linetsky. Pricing equity derivatives subject to bankruptcy. Mathematical Finance, 16(2):255-282, 2006.

[26] L. C. G. Rogers and S. Singh. The cost of illiquidity and its effects on hedging. Mathematical Finance, 20(4):597-615, 2010.

[27] A. Schied and T. Schöneborn. Risk aversion and the dynamics of optimal liquidation strategies in illiquid markets. Finance and Stochastics, 13(2):181-204, 2009.

[28] P. J. Schönbucher. Credit Derivatives Pricing Models: Models, Pricing, Implementation. Wiley Finance, 2003. 